\title{
Overexpression of a panel of cancer stem cell markers enhances the predictive capability of the progression and recurrence in the early stage cholangiocarcinoma
}

Sureerat Padthaisong 1,3, Malinee Thanee, ${ }^{2,3}$, Nisana Namwat 1,2,3, Jutarop Phetcharaburanin 1,2,3, Poramate Klanrit 1,2,3, Narong Khuntikeo 2,3,4, Attapol Titapun²,3,4, Sakkarn Sungkhamanon ${ }^{3,5}$, Hideyuki Saya ${ }^{6}$

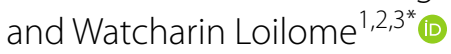

\begin{abstract}
Background: Cancer recurrence is the important problem of cholangiocarcinoma (CCA) patients, lead to a very high mortality rate. Therefore, the identification of candidate markers to predict CCA recurrence is needed in order to effectively manage the disease. This study aims to examine the predictive value of cancer stem cell (CSC) markers on the progression and recurrence of CCA patients.

Methods: The expression of 6 putative CSC markers, cluster of differentiation 44 (CD44), CD44 variant 6 (CD44v6), CD44 variants 8-10 (CD44v8-10), cluster of differentiation 133 (CD133), epithelial cell adhesion molecule (EpCAM), and aldehyde dehydrogenase 1A1 (ALDH1A1), was investigated in 178 CCA tissue samples using immunohistochemistry $(\mathrm{HC})$ and analyzed with respect to clinicopathological data and patient outcome including recurrence-free survival (RFS) and overall survival (OS). The candidate CSC markers were also investigated in serum from CCA patients, and explored for their predictive ability on CCA recurrence.

Results: Elevated protein level of CD44 and positive expression of CD44v6 and CD44v8-10 were significantly associated with short RFS and OS, while high levels of ALDH1A1 were correlated with a favorable prognosis patient. The elevated CD44v6 level was also correlated with higher tumor staging, whereas a decreasing level of ALDH1A1 was correlated with lower tumor staging. The levels of CD44, CD44v6 and CD44v8-10 were also correlated and were associated with a poor outcome. Furthermore, soluble CD44, CD44v6, CD44v8-10 and EpCAM were significantly increased in the recurrence group for early stage CCA; they also correlated with high levels of the tumor marker CA199. Elevated levels of CD44, CD44v6, CD44v8-10 or EpCAM alone or in combination has the potential to predict CCA recurrence.

Conclusions: The overexpression of CD44, CD44v6, CD44v8-10 and EpCAM increases predictability of post-operative CCA recurrence. Moreover, the overexpression of the panel of CSC markers combined with CA19-9 could improve our predictive ability for tumor recurrence in early stage CCA patients. This result may be beneficial for the patients in
\end{abstract}

*Correspondence: watclo@kku.ac.th

1 Department of Biochemistry, Faculty of Medicine, Khon Kaen University,

123 Mittraparp Road, Muang District, Khon Kaen 40002, Thailand

Full list of author information is available at the end of the article

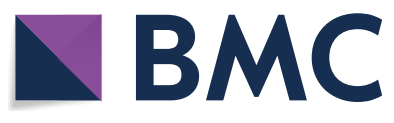

(c) The Author(s) 2020. This article is licensed under a Creative Commons Attribution 4.0 International License, which permits use, sharing, adaptation, distribution and reproduction in any medium or format, as long as you give appropriate credit to the original author(s) and the source, provide a link to the Creative Commons licence, and indicate if changes were made. The images or other third party material in this article are included in the article's Creative Commons licence, unless indicated otherwise in a credit line to the material. If material is not included in the article's Creative Commons licence and your intended use is not permitted by statutory regulation or exceeds the permitted use, you will need to obtain permission directly from the copyright holder. To view a copy of this licence, visit http://creativeco mmons.org/licenses/by/4.0/. The Creative Commons Public Domain Dedication waiver (http://creativecommons.org/publicdomain/ zero/1.0/) applies to the data made available in this article, unless otherwise stated in a credit line to the data. 
order to predict the outcome after treatment and may be useful for clinical intervention in order to improve patient survival.

Keywords: Cholangiocarcinoma, Cancer recurrence, Cancer stem cell marker, Tumor marker, Prognostic factor

\section{Background}

Cholangiocarcinoma (CCA) is the second most common primary hepatic cancer. It originates from the bile duct epithelium, accounting for $10-20 \%$ of primary liver cancers [1]. CCA can be divided into intrahepatic (iCCA), perihilar (pCCA) and distal (dCCA) forms based on their anatomical localization. iCCA arises from the bile duct epithelium inside the liver while pCCA and dCCA arise from epithelium outside of the liver [2]. Surgical resection is the only curative treatment and there is evidence suggesting that surgery with complete resection can improve patient survival [3]. In addition to surgery, adjuvant chemo- or radio-therapy is necessary to improve the patient's outcome [4]. However, to date many patients experience recurrence after surgery resulting in the high mortality rate of CCA patients [5]. Therefore, understanding the tumor biology and the identification of markers to predict cancer recurrence are necessary to manage the disease.

Accumulated evidence suggests that subpopulations of cancer cells, called cancer stem cells (CSCs), show stem cell-like properties such as self-renewal. CSCs play a critical role in many cancer processes, including development, progression and recurrence [6]. Because CSCs impact tumor aggressiveness, CSC markers, which are the markers most commonly expressed in CSCs, become an important factor for predicting cancer progression and recurrence. Currently, several CSC markers have been established in CCA, including cluster of differentiation 44 (CD44), cluster of differentiation 133 (CD133), epithelial cell adhesion molecules (EpCAM), and aldehyde dehydrogenase 1 (ALDH1) [7]. The expression of these markers is usually associated with a poor clinical outcome in patients with different cancers [8-12]. CD44 is a cell surface glycoprotein with a single polypeptide chain. It functions as a cell surface receptor for hyaluronic acid. There are many CD44 variants (CD44v) generated by alternative splicing processes [13]. The expression of CD44 and its variant isoforms relates to tumor progression and recurrence in some cancers [11, 12, 14]. CD133, transmembrane glycoprotein, is another CSC marker for cancer stem-like cells in CCA. High expression of CD133 was reported to be significantly associated with more aggressive tumors and correlated with a worse outcome for cancer patients [15]. Another CSC marker, EpCAM, is mostly overexpressed in tumors of epithelial origin. EpCAM overexpression is usually associated with tumor progression, especially metastasis [16]. Moreover, it has long been recognized that EpCAM can be cleaved [17], and soluble EpCAM is also associated with the aggressive phenotype of tumors [18-20]. Even though surface markers are mostly used to isolate/characterize CSC, other types of markers have also been used to identify and predict tumor progression and patient outcome. ALDH1A1 is an enzyme belonging to the ALDH family that functions as a detoxifying enzyme and also converts retinol (vitamin A) into retinoic acid (RA) [10]. The overexpression of ALDH1A1 is mostly involved in poor cancer prognosis, however, numerous studies suggest that high expression of ALDH1A1 is also associated with a better prognosis of the patients [21]. Although many studies have reported that the expression of CD44, CD44v, CD133, EpCAM and ALDH1A1 is associated with tumor progression and can be used to predict patient's outcome, their prognostic significance in the recurrence of CCA in patients has not been elucidated.

Therefore, in the present study, the expression of the above 6 putative CSC markers was investigated in 178 paraffin-embedded CCA tissue samples using immunohistochemical staining in order to explore their relationship with clinicopathological features and patient survival. Moreover, 4 candidate CSC markers were selected for further experimentation using enzymelinked immunosorbent assay (ELISA) to examine their expression level in serum and to provide a potential CSC panel for predicting of CCA recurrence.

\section{Methods}

\section{Patient selection and follow-up}

Patients diagnosed with CCA and who underwent surgery at Srinagarind Hospital, Khon Kaen University, Khon Kaen, Thailand between February, 2007 and December, 2016 were retrospectively studied. Patients were excluded if they received either radiotherapy or chemotherapy before surgery in order to reduce the effect of neoadjuvant on protein expression. The patients were also excluded if they died within 30 days after surgery to avoid the effect of the operation. Tissue samples and preoperative peripheral blood were obtained from patients and kept in the BioBank of the Cholangiocarcinoma Research Institute. All patients were assessed for clinicopathological characteristics including sex, age, tumor site, histology type, primary tumor ( $\mathrm{T}$ stage), regional lymph node metastasis ( $\mathrm{N}$ stage), distant metastasis ( $\mathrm{M}$ 
stage), TNM stage, and post-operative chemotherapy status. In addition, pre-operative peripheral blood was used for laboratory testing including, tumor markers and liver function test.

For the follow-up protocol after surgery, the patients were examined every 3 months during the first year and every 6 months thereafter. Those patients who developed a new tumor after surgery were defined as having a postoperative recurrence. Overall survival (OS) was defined as the interval from the date of surgery to the time of death or until the last follow-up date, and recurrencefree survival (RFS) was defined as the interval from the date of surgery to the time of recurrence or until the last follow-up date.

\section{Immunohistochemistry (IHC) and grading}

Two independent punctures from each patient were transfer onto a tissue microarray (TMA), and TMA was cut into $4 \mu \mathrm{m}$ sections. IHC staining was performed to investigate protein expression. Tissue sections were deparaffinized and rehydrated with stepwise xylene followed by $100 \%, 90 \%, 80 \%$ and $70 \%$ ethanol. Antigen retrieval was performed by microwave cooking with $10 \mathrm{mM}$ sodium citrate buffer $\mathrm{pH} 6$ and $0.05 \%$ Tween 20 for $10 \mathrm{~min}$. Tissue sections were treated with $0.3 \%$ hydrogen peroxide and $10 \%$ skim milk to block endogenous hydrogen peroxide activity and nonspecific binding for $30 \mathrm{~min}$ of each. Tissue sections was incubated with primary antibodies (CD44; \#ab51037; dilution 1:50, CD44v6; \#ab78960; dilution 1:50, CD133; \#ab19898; dilution 1:100, EpCAM; \#ab71916; dilution 1:100, ALDH1A1; \#ab52492; dilution 1:100) (Abcam, UK), and (CD44v8-10; \#LKG-M001; dilution 1:50) (Cosmo Bio, JP) for $1 \mathrm{~h}$ at room temperature followed by $4{ }^{\circ} \mathrm{C}$ overnight. Sections were washed in phosphate buffered saline (PBS) with $0.1 \%$ tween 20 and incubated with secondary antibody (Dako EnVision) for $1 \mathrm{~h}$, except CD44v8-10 to which was added anti-rat antibody (\#ab6734; (Abcam, UK)) and left for $3 \mathrm{~h}$. The signal was developed with a 3,3'diaminobenzidine tetrahydrochloride (DAB) substrate kit (Vector Laboratories, Inc., CA) for 5-10 min. Sections was counterstained with Mayer's hematoxylin for $2 \mathrm{~min}$ and dehydrated stepwise with $70 \%, 80 \%, 90 \%, 100 \%$ ethanol and xylene. Next, sections were mounted with permount and observed under a light microscope by two independent observers in a blinded manner. Inconsistent data were discussed by the observers until a final agreement was reached.

Protein expression was analyzed according to staining frequency and intensity. The staining frequency of the protein was semi-quantitatively scored based on the percentage of positive cells, $0 \%=$ negative, $1-25 \%=+1$, $26-50 \%=+2$, and $>50 \%=+3$. The intensity of protein expression was scored as weak $=1$, moderate $=2$, and strong $=3$. The final immunohistochemical score was determined by multiplying the intensity scores with the frequency scores, with a minimum score of 0 and a maximum of 9. The average score of each patient was calculated from two independent punctures. Finally, the median value was calculated by grading the scores of all patients. This was used as a cut-off point with the patients having a grading score lower than the median being classified as the low expression group, while those with a grading score equal to or higher than the median were classified as the high expression group [22]. For the proteins which have a median equal to zero, the patients have a grading score equal to zero, being classified as the negative group, while those with a grading score above zero are classified as the positive group.

\section{Enzyme-linked immunosorbent assay (ELISA)}

Indirect ELISA was used to examine soluble protein levels in patient serum. Firstly, serum was diluted in coating buffer (dilution 1:100). Plates were coated with $100 \mu \mathrm{l}$ of serum and incubated at $4{ }^{\circ} \mathrm{C}$ for $18 \mathrm{~h}$. Then, the plates were blocked with $200 \mu \mathrm{l}$ of $5 \%$ skim milk and incubated at $37{ }^{\circ} \mathrm{C}$ for $1 \mathrm{~h}$. After washing, $100 \mu \mathrm{l}$ of primary antibodies (CD44; \#ab2212; dilution 1:200) (Abcam, UK), (CD44v6; AB2080; dilution 1:800) (Merck, Germany), (CD44v8-10; \#LKG-M001; dilution 1:10,000), and (EpCAM; \#MA1-46104; dilution 1:200) (Thermo Fisher Scientific, USA) were added and incubated at $37{ }^{\circ} \mathrm{C}$ for $2 \mathrm{~h}$. Then, the excess antibody was washed off and $100 \mu \mathrm{l}$ of HRP-conjugated secondary antibodies were added (anti-mouse antibody for CD44; \#A16166; dilution 1:400; (Thermo Fisher Scientific, USA), anti-rabbit antibody for CD44v6; \#G21234; dilution 1:1000; (Invitrogen, USA), anti-rat antibody for CD44v8-10, \#ab6734; dilution 1:25,000 (Abcam, UK), anti-mouse antibody for EpCAM, \#A16166, dilution 1:400; (Thermo Fisher Scientific, USA)). After incubation, the excess antibody was washed off and the signal was developed using $100 \mu \mathrm{l}$ of $1 \mathrm{mg} / \mathrm{ml}$ OPD substrate for $30 \mathrm{~min}$. The reaction was stopped with $100 \mu \mathrm{l}$ of $4 \mathrm{~N} \mathrm{H}_{2} \mathrm{SO}_{4}$ reagent and the OD measured on an ELISA reader at $492 \mathrm{~nm}$.

\section{Statistical analyses}

Statistical analyses were carried out using the Statistical Package for the Social Sciences; SPSS software v.17. The association between protein expression and the clinicopathological features of the CCA patients was determined using the Chi square test. Overall and recurrence-free survival analyses were performed using Kaplan-Meier (log-rank) analysis. The correlation between protein types was analyzed using Pearson's correlation. The different of IHC score in different staging was analyzed 
using Kruskal-Wallis Test. The results from ELISA were analyzed by student's $t$ test. The receiver operator characteristic curve (ROC curve) and logistic regression were used to determine the predictive ability with respect to cancer recurrence of soluble protein levels or the combination with tumor markers. A $p$-value less than 0.05 was considered as statistically significant.

\section{Results}

\section{Characteristics of CCA patients}

A total of 178 CCA samples were studied with 64 cases (36\%) from females and 114 (64\%) from males. The age ranged between 42 and 82 years (median $=61$ years). 96 cases $(54 \%)$ were intrahepatic CCA and $82(46 \%)$ were extrahepatic CCA. The histology type was characterized as papillary 83 cases (47\%) and other 95 cases (53\%). The staging was classified according to TNM staging. 109 cases $(61 \%)$ were classified as primary tumor $(\mathrm{T})$ stage $\mathrm{I}$, II and 69 cases (39\%) were T stage III, IV. Regional lymph nodes $(\mathrm{N})$ and distant $(\mathrm{M})$ metastases were also characterized. Among 178 patients, 72 cases (40\%) had regional lymph node metastasis but only 4 cases $(2 \%)$ showed distant metastasis. 80 cases (45\%) were divided into early stage (TNM stage I, II) while 98 cases (55\%) were late or advanced stage (TNM stage III, IV) (Table 1).

\section{Correlation between CSC marker expression and clinicopathological features}

The expression levels of the CSC marker were investigated using immunohistochemistry. The representative figures of a normal bile duct, the precancerous (dysplasia) stage and CCA are shown in Fig. 1. To investigate the correlation between protein expression and clinicopathological features, the expression of CSC markers was divided into those with low and those with high expression and also those with negative and those with positive expression. High expression of candidate CSC markers CD44, EpCAM, ALDH1A1 and positive expression of CD44v6, CD44v8-10, and CD133 were $65 \%, 52 \%$, $47 \%, 38 \%, 42 \%$, and $36 \%$, respectively. High expression of CD44 and positive expression of CD44v6 was observed mostly in males $(p=0.028$ and $p=0.026)$. In addition,

Table 1 Clinicopathological characteristics of CCA patients and the correlation with 6 putative CSC marker expression

\begin{tabular}{|c|c|c|c|c|c|c|c|c|c|c|c|c|c|c|c|c|c|c|c|}
\hline \multirow[t]{2}{*}{ Variables } & \multirow[t]{2}{*}{ n (178) } & \multicolumn{2}{|c|}{ CD44 } & \multirow[t]{2}{*}{$p$} & \multicolumn{2}{|c|}{ CD44v6 } & \multirow[t]{2}{*}{$p$} & \multicolumn{2}{|c|}{$\begin{array}{l}\text { CD44v8- } \\
10\end{array}$} & \multirow[t]{2}{*}{$p$} & \multicolumn{2}{|c|}{ CD133 } & \multirow[t]{2}{*}{$p$} & \multicolumn{2}{|c|}{ EpCAM } & \multirow[t]{2}{*}{$p$} & \multicolumn{2}{|c|}{ ALDH1A1 } & \multirow[t]{2}{*}{$p$} \\
\hline & & Low & High & & Neg. & Pos. & & Neg. & Pos. & & Neg. & Pos. & & Low & High & & Low & High & \\
\hline \multicolumn{20}{|l|}{ Sex } \\
\hline Female & 64 & 29 & 35 & & 33 & 31 & & 31 & 33 & & 37 & 27 & & 28 & 36 & & 34 & 30 & \\
\hline Male & 114 & 33 & 81 & 0.028 & 78 & 36 & 0.026 & 72 & 42 & 0.056 & 77 & 37 & 0.194 & 57 & 57 & 0.423 & 60 & 54 & 0.950 \\
\hline \multicolumn{20}{|l|}{ Age (year) } \\
\hline Less than 61 & 85 & 33 & 52 & & 54 & 31 & & 49 & 36 & & 54 & 31 & & 44 & 41 & & 43 & 42 & \\
\hline 61 or greater & 93 & 29 & 64 & 0.285 & 57 & 36 & 0.758 & 54 & 39 & 0.955 & 60 & 33 & 0.891 & 41 & 52 & 0.306 & 51 & 42 & 0.570 \\
\hline \multicolumn{20}{|l|}{ Tumor site } \\
\hline Intrahepatic & 96 & 33 & 63 & & 49 & 47 & & 52 & 44 & & 54 & 42 & & 40 & 56 & & 48 & 48 & \\
\hline Extrahepatic & 82 & 29 & 53 & 0.890 & 62 & 20 & 0.001 & 51 & 31 & 0.280 & 60 & 22 & 0.019 & 45 & 37 & 0.079 & 46 & 36 & 0.417 \\
\hline \multicolumn{20}{|l|}{ Histology type } \\
\hline Papillary & 83 & 32 & 51 & & 53 & 30 & & 46 & 37 & & 55 & 28 & & 37 & 46 & & 40 & 43 & \\
\hline Others & 95 & 30 & 65 & 0.330 & 58 & 37 & 0.700 & 57 & 38 & 0.537 & 59 & 36 & 0.564 & 48 & 47 & 0.428 & 54 & 41 & 0.249 \\
\hline \multicolumn{20}{|c|}{ Primary tumor ( $\mathrm{T}$ ) } \\
\hline I, II & 109 & 44 & 65 & & 75 & 34 & & 65 & 44 & & 70 & 39 & & 51 & 58 & & 53 & 56 & \\
\hline III, IV & 69 & 18 & 51 & 0.051 & 36 & 33 & 0.026 & 38 & 31 & 0.548 & 34 & 25 & 0.951 & 34 & 35 & 0.746 & 41 & 28 & 0.160 \\
\hline \multicolumn{20}{|c|}{ Lymph nodes (N) metastasis } \\
\hline No & 106 & 39 & 67 & & 76 & 30 & & 66 & 40 & & 68 & 38 & & 52 & 54 & & 45 & 61 & \\
\hline Yes & 72 & 23 & 49 & 0.505 & 35 & 37 & 0.002 & 37 & 35 & 0.149 & 46 & 26 & 0.971 & 33 & 39 & 0.673 & 49 & 23 & 0.001 \\
\hline \multicolumn{20}{|c|}{ Distant metastasis (M) } \\
\hline No & 174 & 59 & 115 & & 107 & 67 & & 102 & 72 & & 112 & 62 & & 83 & 91 & & 90 & 84 & \\
\hline Yes & 4 & 3 & 1 & 0.088 & 4 & 0 & 0.116 & 1 & 3 & 0.178 & 2 & 2 & 0.554 & 2 & 2 & 0.927 & 4 & 0 & 0.056 \\
\hline \multicolumn{20}{|l|}{ TNM stage } \\
\hline$|| \mid$, & 80 & 32 & 48 & & 59 & 21 & & 51 & 29 & & 51 & 29 & & 40 & 40 & & 32 & 48 & \\
\hline III, IV & 98 & 30 & 68 & 0.191 & 52 & 46 & 0.005 & 52 & 46 & 0.151 & 63 & 35 & 0.941 & 45 & 53 & 0.588 & 62 & 36 & 0.002 \\
\hline
\end{tabular}

CD44 cluster of differentiation 44, CD44v CD44 variant, CD133 cluster of differentiation 133, EpCAM epithelial cell adhesion molecules, ALDH1A1 aldehyde dehydrogenase 1A1, TNM primary tumor-node-metastasis, Neg negative expression, Pos positive expression 


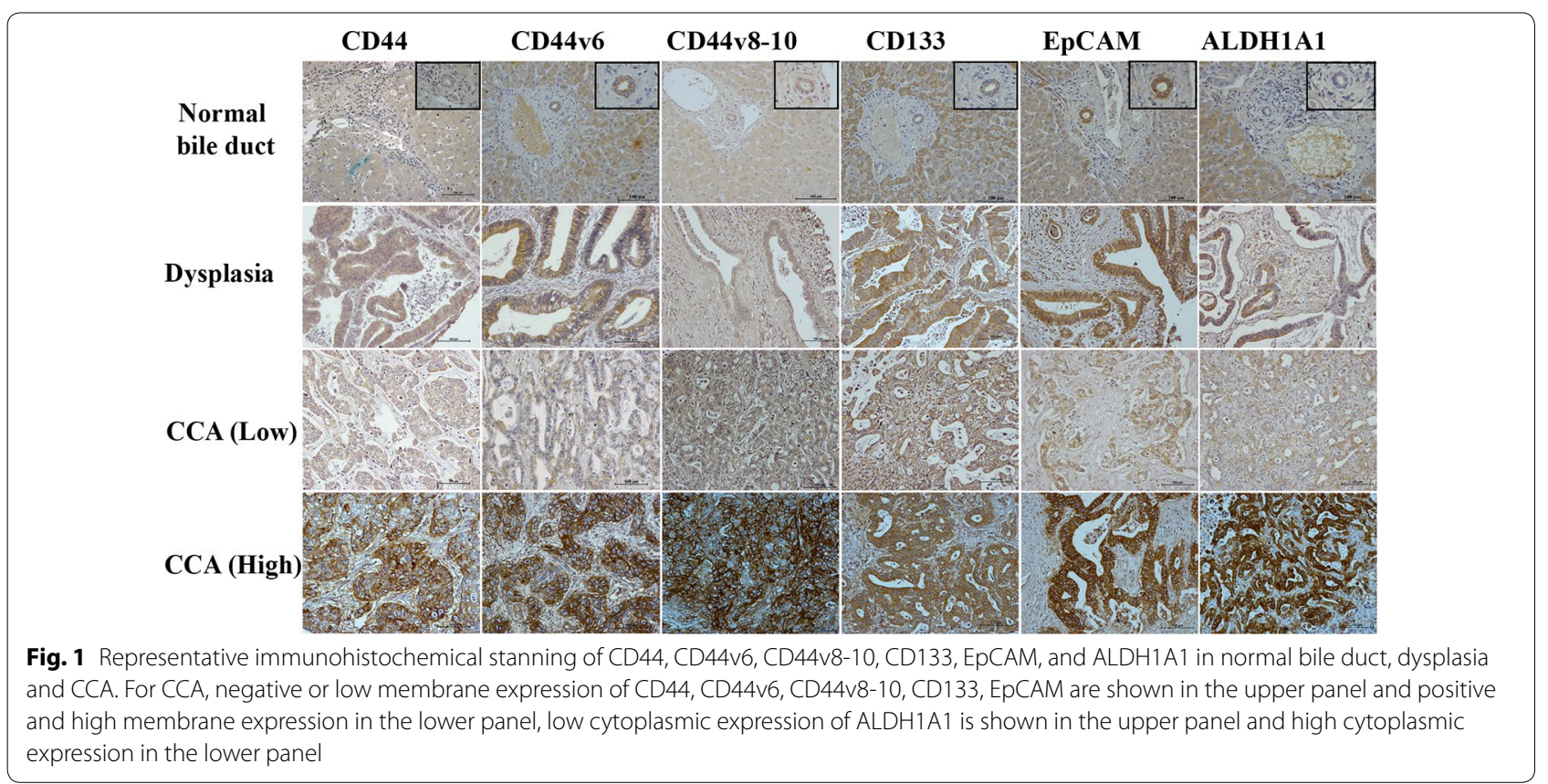

positive CD44v6 and CD133 expression was frequently observed in intrahepatic CCA $(p=0.001$ and $p=0.019)$. A significant association was found between $\mathrm{T}$ stage and CD44v6 expression ( $p=0.026)$. Additionally, reginal lymph node metastasis and TNM staging were significantly associated with CD44v6 ( $p=0.002$ and $p=0.005)$ and ALDH1A1 expression $(p=0.001$ and $p=0.002)$ (Table 1).

\section{The prognostic significance of clinicopathological features} To identify prognostic factors for CCA patients, we analyzed all clinicopathological features including sex, age, tumor site, histology type, $\mathrm{T}$ stage, regional lymph node metastasis and distant metastasis status, TNM stage, post-operative chemotherapy (CMT) status with recurrence-free survival (RFS) and overall survival (OS) of the patients. The median RFS and OS were 15 and 17 months, respectively. Among all clinicopathological features, we found that patients with a higher $\mathrm{T}$ stage, regional lymph nodes and higher TNM staging were significantly correlated with shorter RFS compared with patients with a low $\mathrm{T}$ stage, absent regional lymph nodes, or low TNM stage $(p<0.001, p=0.001$ and $p<0.001$, respectively). The results for OS analysis also showed a similar result, except that patients with age equal to 61 or greater were also significantly associated with a short OS $(p=0.032)$. There was no significant correlation between sex, histology type, tumor site, distant metastasis status, and post-operative CMT status with RFS and OS (Table 2).

\section{The expression of CSC markers and their prognostic} significance in CCA patients

The expression of candidate CSC markers CD44, CD44v6, CD44v8-10, CD133, EpCAM, and ALDH1A1 was analyzed with respect to RFS and OS. Univariate analysis showed that the patients with a high expression of CD44, positive expression of CD44v6 and CD44v810 had a shorter RFS compared with other patients $(p=0.007, p=0.001$ and $p=0.007$, respectively). In addition, a high expression of CD44 and ALDH1A1, positive expression of CD44v6 and CD44v8-10 was associated with a shorter OS compared with the other group of patients $(p=0.001, p=0.022, p=0.006$ and $p<0.001$, respectively) (Table 2). Moreover, multivariate analysis showed that CD44 and CD44v8-10 could be used as prognostic factors independent of clinicopathological characteristics for RFS $(p=0.020$ and $p=0.012)$ (Table 3$)$ and $\mathrm{OS}(p=0.002$ and $p=0.001)$ (Table 4$)$.

Kaplan-Meier analysis was used to examine the importance of tumor location. The result from intrahepatic CCA showed that a high expression of CD44 or the positive expression of CD44v6, and CD44v8-10 was significantly correlated with a shorter RFS compared with samples showing a low expression $(p=0.007, p=0.017$ and $p<0.001$, respectively), while a high expression of EpCAM and ALDH1A1 was significantly correlated with a favorable prognosis in patients $(p=0.028$ and $p=0.008$ ) (Fig. 2). The results from OS analysis showed that patients with a high expression of CD44 or a positive expression of CD44v8-10 or a low expression of 
Table 2 Univariate analysis of factors predicting recurrence-free and overall survival

\begin{tabular}{|c|c|c|c|c|c|c|}
\hline \multirow[t]{2}{*}{ Variable } & \multirow{2}{*}{$\begin{array}{l}\text { Median RFS } \\
\text { (months) }\end{array}$} & \multicolumn{2}{|c|}{ Recurrence-free survival } & \multirow{2}{*}{$\begin{array}{l}\text { Median } \\
\text { OS (months) }\end{array}$} & \multicolumn{2}{|c|}{ Overall survival } \\
\hline & & HR & $P(95 \% \mathrm{Cl})$ & & HR & $P(95 \% \mathrm{Cl})$ \\
\hline Overall & 15.0 & & & 17.0 & & \\
\hline \multicolumn{7}{|l|}{ Sex } \\
\hline Female & 13.0 & 1 & $0.336(0.853-1.593)$ & 16.9 & 1 & $0.270(0.872-1.634)$ \\
\hline Male & 15.0 & 1.166 & & 17.4 & 1.193 & \\
\hline \multicolumn{7}{|l|}{ Age (year) } \\
\hline Less than 61 & 16.0 & 1 & $0.208(0.899-1.630)$ & 19.2 & 1 & $0.032(1.028-1.876)$ \\
\hline 61 or greater & 13.0 & 1.211 & & 16.3 & 1.388 & \\
\hline \multicolumn{7}{|l|}{ Tumor site } \\
\hline Intrahepatic & 12.5 & 1 & 0.449 (0.664-1.199) & 16.0 & 1 & $0.560(0.680-1.232)$ \\
\hline Extrahepatic & 16.0 & 0.892 & & 18.5 & 0.915 & \\
\hline \multicolumn{7}{|l|}{ Histology type } \\
\hline Papillary & 16.0 & 1 & $0.253(0.884-1.599)$ & 17.6 & 1 & $0.759(0.779-1.409)$ \\
\hline Others & 13.0 & 1.189 & & 16.3 & 1.047 & \\
\hline \multicolumn{7}{|c|}{ Primary tumor $(\mathrm{T})$} \\
\hline $1, \|$ & 18.0 & 1 & $<0.001(1.956-3.771)$ & 22.0 & 1 & $<0.001(1.907-3.629)$ \\
\hline III, IV & 9.0 & 2.716 & & 11.0 & 2.630 & \\
\hline \multicolumn{7}{|c|}{ Reginal lymph nodes $(\mathrm{N})$ metastasis } \\
\hline No & 17.0 & 1 & $0.001(1.265-2.362)$ & 20.0 & 1 & $<0.001(1.442-2.704)$ \\
\hline Yes & 10.5 & 1.729 & & 13.0 & 1.975 & \\
\hline \multicolumn{7}{|c|}{ Distant metastasis (M) } \\
\hline No & 15.0 & 1 & $0.762(0.432-3.150)$ & 17.0 & 1 & $0.519(0.513-3.751)$ \\
\hline Yes & 12.0 & 1.166 & & 13.5 & 1.387 & \\
\hline \multicolumn{7}{|l|}{ TNM stage } \\
\hline I, II & 18.0 & 1 & $<0.001(1.576-2.986)$ & 21.6 & 1 & $<0.001(1.711-3.255)$ \\
\hline $\mathrm{III}, \mathrm{IV}$ & 10.0 & 2.169 & & 12.8 & 2.360 & \\
\hline \multicolumn{7}{|c|}{ Post-operative CMT } \\
\hline No & 15.0 & 1 & $0.933(0.727-1.340)$ & 16.9 & 1 & $0.556(0.671-1.239)$ \\
\hline Yes & 15.0 & 0.987 & & 19.0 & 0.912 & \\
\hline \multicolumn{7}{|l|}{ CD44 } \\
\hline Low & 18.0 & 1 & $0.007(1.129-2.170)$ & 22.0 & 1 & $0.001(1.250-2.399)$ \\
\hline High & 13.0 & 1.565 & & 16.1 & 1.732 & \\
\hline \multicolumn{7}{|l|}{ CD44v6 } \\
\hline Neg. & 17.0 & 1 & $0.001(1.234-2.311)$ & 19.2 & 1 & $0.006(1.128-2.081)$ \\
\hline Pos. & 11.0 & 1.689 & & 12.8 & 1.532 & \\
\hline \multicolumn{7}{|l|}{ CD44v8-10 } \\
\hline Neg. & 16.0 & 1 & $0.007(1.125-2.072)$ & 20.0 & 1 & $<0.001(1.271-2.352)$ \\
\hline Pos. & 13.0 & 1.527 & & 14.1 & 1.729 & \\
\hline \multicolumn{7}{|l|}{ CD133 } \\
\hline Neg. & 16.0 & 1 & $0.103(0.949-1.765)$ & 18.1 & 1 & $0.083(0.965-1.794)$ \\
\hline Pos. & 12.5 & 1.295 & & 16.1 & 1.316 & \\
\hline \multicolumn{7}{|l|}{ EpCAM } \\
\hline Low & 14.0 & 1 & $0.135(0.594-1.073)$ & 16.3 & 1 & $0.261(0.629-1.134)$ \\
\hline High & 16.0 & 0.798 & & 18.6 & 0.844 & \\
\hline \multicolumn{7}{|l|}{$\mathrm{ALDH} 1 \mathrm{~A} 1$} \\
\hline Low & 11.0 & 1 & $0.075(0.569-1.028)$ & 16.0 & 1 & $0.022(0.525-0.950)$ \\
\hline High & 17.0 & 0.765 & & 19.9 & 0.706 & \\
\hline
\end{tabular}

TNM primary tumor-node-metastasis, post-operative, CMT post-operative chemotherapeutic treatment, $H R$ hazard ratio, 95\% Cl $95 \%$ confidence interval, CD44 cluster of differentiation 44, CD44V CD44 variant, CD133 cluster of differentiation 133, EpCAM epithelial cell adhesion molecules, ALDH1A1 aldehyde dehydrogenase 1A1, TNM primary tumor-node-metastasis, Neg negative expression, Pos positive expression 
Table 3 Multivariate analysis of factors predicting recurrence-free survival

\begin{tabular}{lllr}
\hline Variable & \multicolumn{3}{c}{ Recurrence-free survival } \\
\cline { 2 - 4 } & HR & $\mathbf{9 5 \%} \mathbf{C l}$ & \multicolumn{1}{c}{$\boldsymbol{p}$} \\
\hline Model A & & & \\
$\quad$ Primary tumor (T) (III/IV vs I/II) & 2.404 & $1.537-3.759$ & $<0.001$ \\
Lymph nodes metastasis (N) (Yes vs No) & 1.451 & $0.944-2.229$ & 0.090 \\
TNM Stage (III/IV vs I/II) & 1.025 & $0.589-1.783$ & 0.931 \\
CD44 (High vs Low) & 1.476 & $1.062-2.051$ & 0.020 \\
Model B & & & \\
Primary tumor (T) (III/IV vs I/II) & 2.341 & $1.482-3.697$ & $<0.001$ \\
Lymph nodes metastasis (N) (Yes vs No) & 1.368 & $0.880-2.126$ & 0.164 \\
TNM Stage (III/IV vs I/II) & 1.024 & $0.579-1.871$ & 0.934 \\
CD44v6 (Pos. vs Neg.) & 1.350 & $0.975-1.871$ & 0.071 \\
Model C & & & \\
Primary tumor (T) (III/IV vs I/II) & 2.665 & $1.686-4.212$ & $<0.001$ \\
Lymph nodes metastasis (N) (Yes vs No) & 1.441 & $0.934-2.223$ & 0.098 \\
TNM Stage (III/IV vs I/II) & 0.933 & $0.528-1.649$ & 0.810 \\
CD44v8-10 (Pos. vs Neg.) & 1.491 & $1.092-2.036$ & 0.012 \\
\hline
\end{tabular}

TNM size of primary tumor-node metastasis-distant metastasis, CD44 cluster of differentiation 44, CD44v CD44 variant, Neg negative expression, Pos positive expression

ALDH1A1 also had a shorter OS compared with other groups ( $p=0.002, p<0.001$ and $p=0.002$, respectively) (Fig. 2). The result from extrahepatic CCA showed that a positive expression of CD44v6 was significantly correlated with a shorter RFS and OS ( $p=0.034$ and $p=0.039)$ (Fig. 3). Additionally, a positive expression of CD133 was significantly correlated with a shorter OS compared with samples showing a low expression $(p=0.033)$ (Fig. 3).

In addition to the DFS and OS analyses, the differences in IHC scores for different protein types were evaluated for different tumor stages. The expression of CD44, CD44v6, and CD44v8-10 seems to increase at higher stages compared with stage I tumor (Fig. $4 \mathrm{a}-\mathrm{c}$ ). Significant differences were observed between stages I and IV, II and IV of CD44v6 $(p=0.033$ and $p=0.020$, respectively) (Fig. 4b). In addition, the expression level of ALDH1A1 could be used to classify tumor staging. We found that ALDH1A1 expression level decreased along with tumor staging and was significantly decreased in stages III and IV compared with stage I tumor ( $p=0.019$ and $p=0.013$ ) (Fig. $4 \mathrm{f}$ ).

CD44, CD44v6, CD44v8-10 and ALDH1A1 showed prognostic significance for CCA patients. The correlation between these markers was therefore further analyzed and significant positive correlations between CD44, CD44v6, and CD44v8-10 were observed, while there was no significant correlation between ALDH1A1 with the other markers (Table 5). The combination of
Table 4 Multivariate analysis of factors predicting overall survival

\begin{tabular}{|c|c|c|c|}
\hline \multirow[t]{2}{*}{ Variable } & \multicolumn{3}{|c|}{ Overall survival } \\
\hline & HR & $95 \% \mathrm{Cl}$ & $p$ \\
\hline \multicolumn{4}{|l|}{ Model A } \\
\hline Age less (61 or greater vs than 61$)$ & 1.429 & $1.051-1.944$ & 0.023 \\
\hline Primary tumor (T) (III/IV vs I/II) & 2.370 & $1.517-3.702$ & $<0.001$ \\
\hline Lymph nodes metastasis (N) (Yes Vs No) & 1.753 & $1.129-2.723$ & 0.012 \\
\hline TNM stage (III/IV vs I/II) & 1.013 & $0.576-1.782$ & 0.964 \\
\hline CD44 (high vs low) & 1.701 & $1.221-2.371$ & 0.002 \\
\hline \multicolumn{4}{|l|}{ Model B } \\
\hline Age less (61 or greater vs than 61) & 1.510 & $1.113-2.050$ & 0.008 \\
\hline Primary tumor (T) (III/IV vs I/II) & 2.321 & $1.474-3.654$ & $<0.001$ \\
\hline Lymph nodes metastasis (N) (Yes vs No) & 1.706 & $1.086-2.682$ & 0.021 \\
\hline TNM stage (III/IV vs I/II) & 0.987 & $0.554-1.759$ & 0.964 \\
\hline CD44v6 (Pos. vs Neg.) & 1.168 & $0.848-1.609$ & 0.340 \\
\hline \multicolumn{4}{|l|}{ Model C } \\
\hline Age less (61 or greater vs than 61) & 1.493 & $1.100-2.027$ & 0.010 \\
\hline Primary tumor (T) (III/IV vs I/II) & 2.765 & $1.739-4.395$ & $<0.001$ \\
\hline Lymph nodes metastasis (N) (yes vs no) & 1.818 & $1.167-2.833$ & 0.008 \\
\hline TNM Stage (III/IV vs I/II) & 0.827 & $0.461-1.485$ & 0.525 \\
\hline CD44v8-10 (Pos. vs Neg.) & 1.694 & $1.234-2.326$ & 0.001 \\
\hline \multicolumn{4}{|l|}{ Model E } \\
\hline Age less (61 or greater vs than 61) & 1.509 & $1.112-2.047$ & 0.008 \\
\hline Primary tumor (T) (III/IV vs I/II) & 2.411 & $1.545-3.764$ & $<0.001$ \\
\hline Lymph nodes metastasis (N) (yes vs no) & 1.724 & $1.104-2.691$ & 0.017 \\
\hline TNM stage (III/IV vs I/II) & 0.955 & $0.539-1.693$ & 0.875 \\
\hline ALDH1A1 (high vs low) & 0.867 & $0.636-1.182$ & 0.366 \\
\hline
\end{tabular}

TNM size of primary tumor-node metastasis-distant metastasis, CD44 cluster of differentiation 44, CD44v CD44 variant, ALDH1A1 aldehyde dehydrogenase 1A1, Neg negative expression, $P$ os positive expression

high expression of CD44 with positive expression of CD44v6 and CD44v8-10 was significantly associated with RFS $(p=0.001$ and $p=0.002)$ and OS $(p=0.001$ and $p<0.001)$ in intrahepatic CCA. Patients with high or positive expression of two or three markers had a poorer prognosis compared with other groups of patients (Fig. 5a and 5b). On the other hand, only high expression of CD44 with a positive expression of CD44v6 and CD44v8-10 was significantly associated with OS ( $p=0.016)$ (Fig. 6b).

\section{The correlation of soluble CSC markers with cancer recurrence}

The previous results showed that CD44, CD44v6, and CD44v8-10 were significantly correlated with RFS. In order to identify soluble CSC markers that can be used to predict cancer relapse, soluble CD44, CD44v6, and CD44v8-10 were further determined in CCA sera. Moreover, soluble EpCAM was also investigated because there is considerable evidence suggesting that 


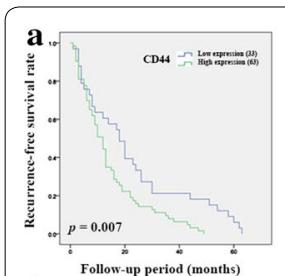

d

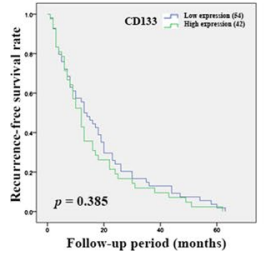

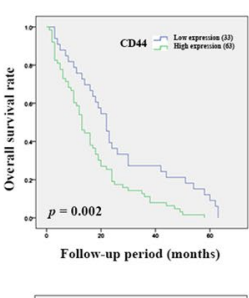
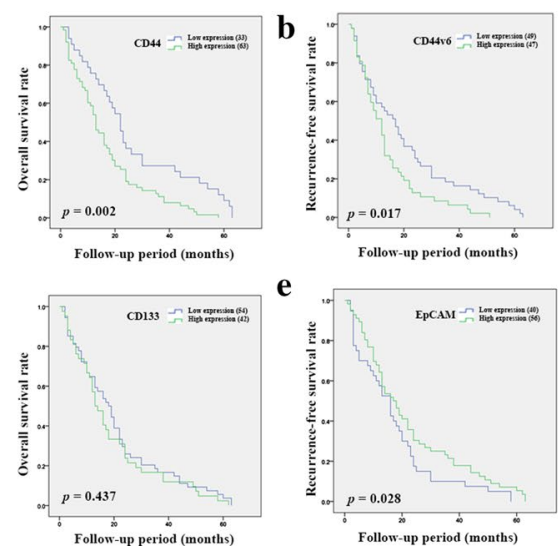

e
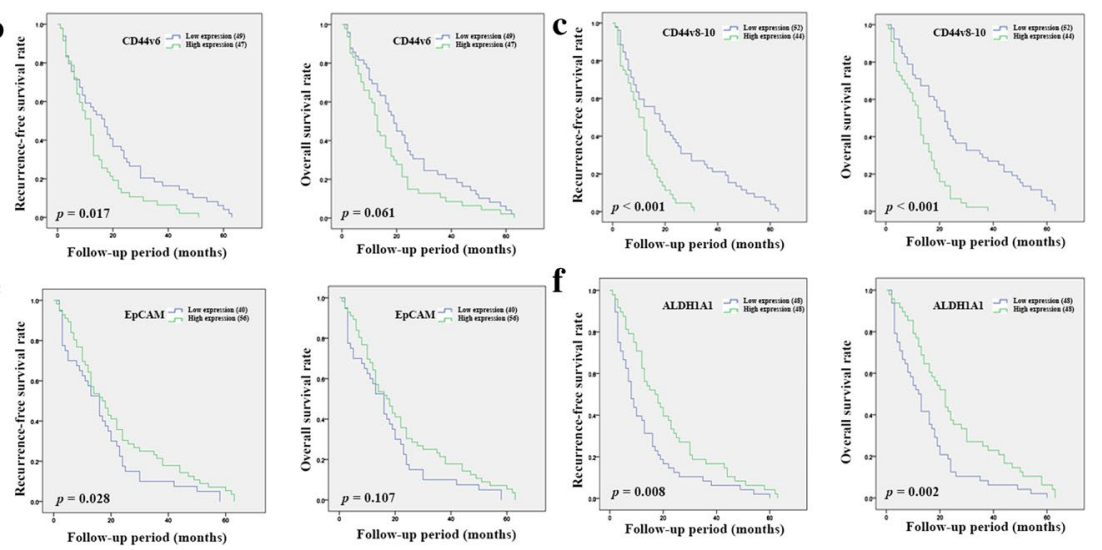
in intrahepatic CCA patients

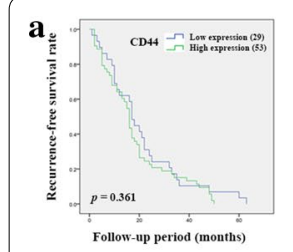

d

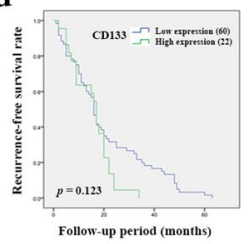

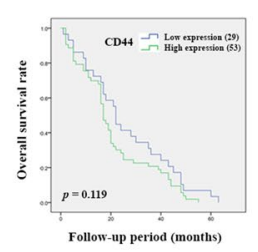

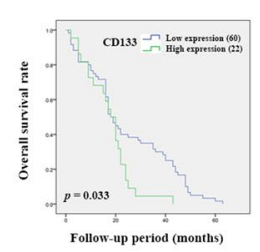

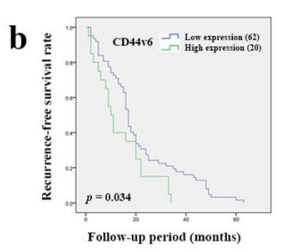

e

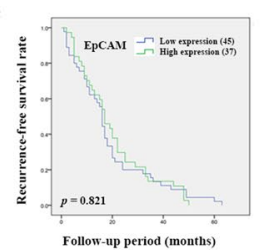

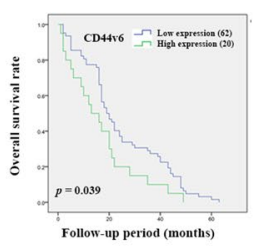
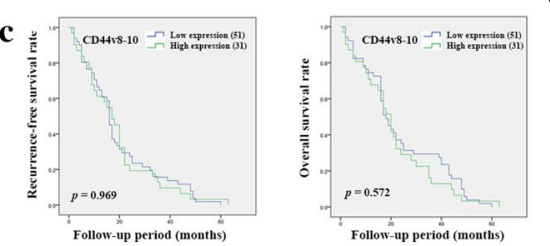

f
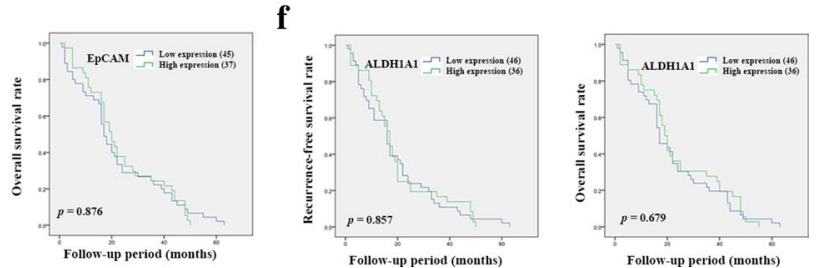

Fig. 3 Kaplan-Meier analyses of recurrence-free and overall survival of CD44 (a), CD44v6 (b), CD44v8-10 (c), CD133 (d), EpCAM (e), and ALDH1A1 (f) in extrahepatic CCA patients

this plays an important role in the progression of many cancers. In addition, the result of IHC showed that T stage, the present of lymph node metastasis and TNM staging were associated with RFS and OS. Therefore, the different of soluble CSC markers, CD44, CD44v6, CD44v8-10 and EpCAM on patients with and without recurrence was analyzed according to tumor staging in order to avoid the effect of T, N and TNM stage on recurrence. The detailed information of 127 sera CCA samples was summarized in Additional file 1: Table S1. The result showed that patients with early stage CCA had levels of soluble CSC markers, CD44, CD44v6, CD44v8-10, and EpCAM that were significantly increased in patients with recurrence $(p=0.019$, $p=0.028, p=0.031$, and $p=0.001)$. On the other hand, there were no differences in soluble CSC markers between patients with recurrence and those without recurrence in the late stage (Fig. 7).

\section{Correlation between CSC marker levels in sera} with clinicopathological features and laboratory results The correlation between the levels of soluble CSC markers with clinicopathological features and laboratory results was analyzed. The results from the early stage group show that high levels of CD44, CD44v8-10 and EpCAM were significantly correlated with high levels of CA19-9 ( $p=0.006, p=0.011$ and $p<0.001$, respectively) (Table 6). On the other hand, there was no correlation found between sex, age, tumor site, histology type, CEA, AFP and liver function test. In addition, the results from the late stage group show that a high level of CD44v6 was significantly associated with elevated total bilirubin, direct bilirubin, AST and ALP $(p=0.037, p=0.029$, $p=0.037$ and $p=0.049$, respectively) (Table 7). Moreover, CD44v8-10 and EpCAM were also associated with elevated of ALP $(p=0.024$ and $p=0.006)$ (Table 7). 

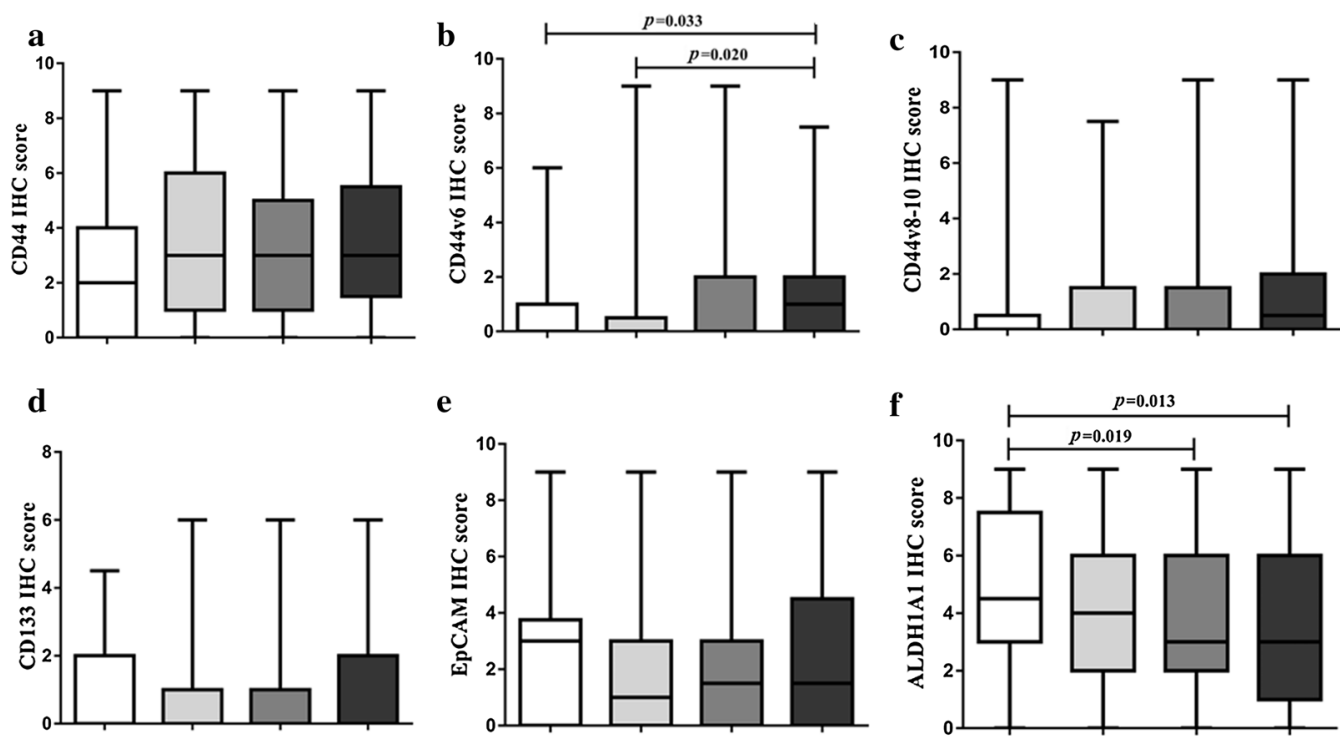

e

f
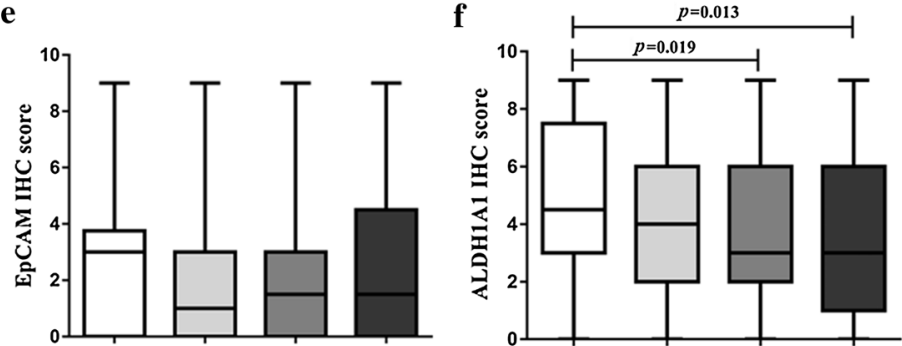

Stage I ( $\mathrm{n}=37)$

Stage II ( $\mathrm{n}=43)$

Stage III ( $=51)$

Stage IV $(\mathrm{n}=47)$

Fig. 4 The different IHC scores for the various protein types, CD44 (a), CD44v6 (b), CD44v8-10 (c), CD133 (d), EpCAM (e), and ALDH1A1 (f) according to tumor staging

Table 5 The correlation coefficients between immunohistochemistry result of CD44, CD44v6, CD44v8-10, and ALDH1A1 in human CCA tissues

\begin{tabular}{|c|c|c|c|c|c|}
\hline & & CD44 & CD44v6 & CD44v8-10 & ALDH1A1 \\
\hline \multirow[t]{2}{*}{ CD44 } & Correlation coefficient & 1 & 0.203 & 0.170 & -0.018 \\
\hline & $p$ & & 0.007 & 0.023 & 0.816 \\
\hline \multirow[t]{2}{*}{ CD44v6 } & Correlation coefficient & & 1 & 0.394 & -0.014 \\
\hline & $p$ & & & $<0.001$ & 0.849 \\
\hline \multirow[t]{2}{*}{ CD44v8-10 } & Correlation coefficient & & & 1 & -0.077 \\
\hline & $p$ & & & & 0.849 \\
\hline \multirow[t]{2}{*}{ ALDH1A1 } & Correlation coefficient & & & & 1 \\
\hline & $p$ & & & & \\
\hline
\end{tabular}

CD44 cluster of differentiation 44, CD44v CD44 variant, ALDH1A1 aldehyde dehydrogenase $1 \mathrm{~A} 1$

Predictive value of soluble CSC markers for post-operative recurrence

The receiver operator characteristic (ROC) curve was analyzed according tumor staging. The cut-off values for soluble CD44, CD44v6, CD44v8-10 and EpCAM suitable for the discrimination between recurrence and nonrecurrence in the patients with early stage CCA were 0.505 (area under curve; $\mathrm{AUC}=0.670, p=0.029$ ), 0.814 $(\mathrm{AUC}=0.670, p=0.029), 0.713(\mathrm{AUC}=0.702, p=0.010)$, and 0.506 (AUC $=0.739, p=0.002$ ), respectively (Additional file 2: Fig. S1). The sensitivity and specificity to distinguish between recurrence and non-recurrence are shown in Table 8 . The positive predictive value (PPV) and negative predictive value (NPV) were 51.7 and 77.4 for CD44, 55.6 and 78.8 for CD44v6, 59.3 and 81.8 for CD44v8-10 and 62.5 and 80.6 for EpCAM. By using the cut-off derived from the ROC curve, the predictive ability of CD44, CD44v6, CD44v8-10 and EpCAM on post-operative recurrence was explored. The crude and adjusted odds ratio (OR) of CD44, CD44v6, CD44v8-10 and EpCAM were $3.67(p=0.022), 4.64(p=008), 6.55$ $(p=0.002), 6.91(p=0.001)$ and $3.62(p=0.031), 4.98$ $(p=0.009), 5.92(p=0.004), 6.23(p=0.003)$ (Table 9). On the other hand, soluble CD44, CD44v6, CD44v8-10, 

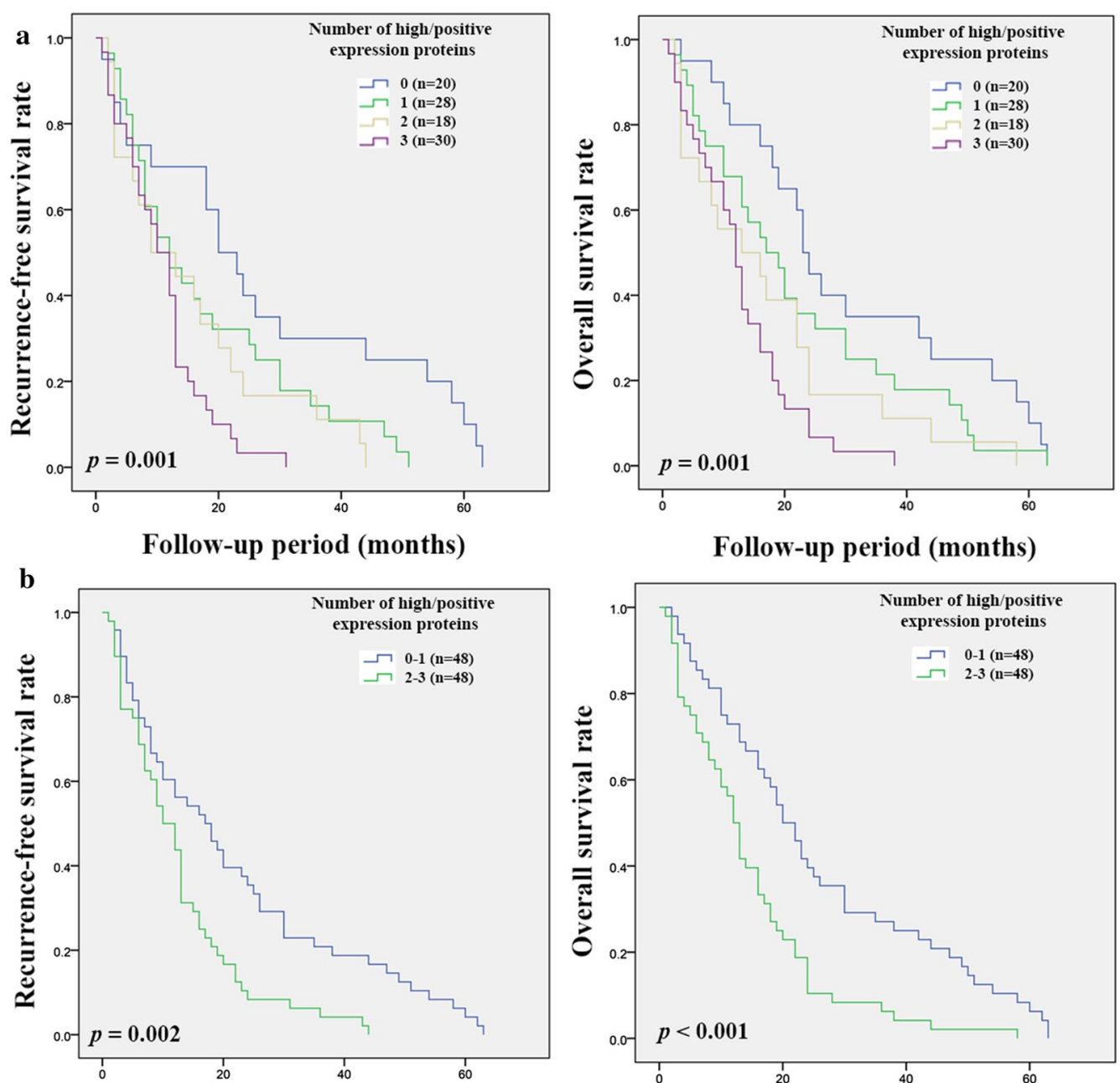

Follow-up period (months)

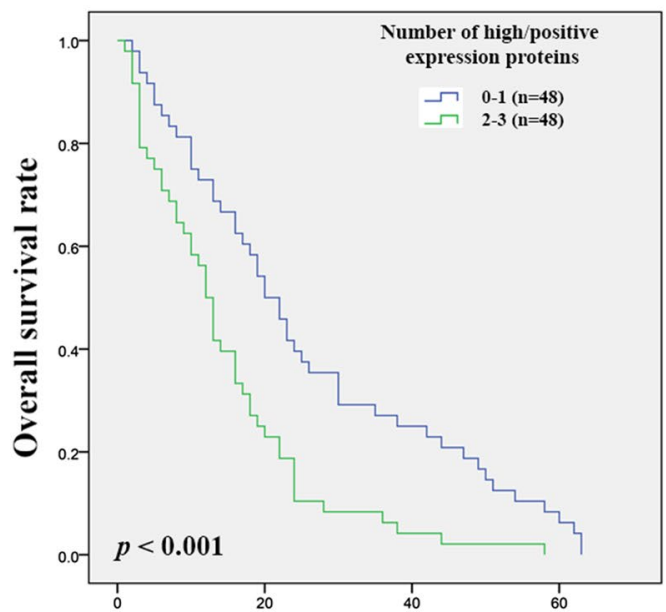

Follow-up period (months)

Follow-up period (months)

Fig. 5 Kaplan-Meier analyses of recurrence-free and overall survival on the combination of CD44, CD44v6, and CD44v8-10 expression in intrahepatic CCA patients. a Recurrence-free and overall survival of patients according to the number of high or positive expression proteins. $\mathbf{b}$ Recurrence-free and overall survival of patients with zero or one high or positive expression marker versus high or positive expression of two or three markers

and EpCAM were not suitable to distinguish between recurrence and non-recurrence in patients with late stage CCA (Additional file 3: Fig. S2).

\section{The combination of soluble CSC markers and CA19-9 for improving predictive ability for post-operative recurrence}

Soluble CD44, CD44v6, CD44v8-10 and EpCAM are promising factors for predicting cancer recurrence. A combination of these markers and their predictive efficacy for cancer recurrence was further examined. Interestingly, a combination of high levels of CD44, CD44v6, CD44v8-10 and EpCAM could increase the risk for recurrence with a high value of crude OR (crude OR $=7.08, p=0.004$ ) and adjusted OR (adjusted $\mathrm{OR}=7.39, p=0.006)$. Moreover, the best predictive ability for recurrence was observed with the combination of high expression of these 4 CSC markers and elevated CA19-9 levels with an increase of the crude and adjusted OR to $12.25(p=0.005)$ and $15.28(p=0.011)$, respectively (Table 10). The survival analysis was also evaluated in patients with high levels of CD44, CD44v6, CD44v8-10 and EpCAM combined with an elevated CA19-9 level compared with other groups of patients. Patients with high levels of CD44, CD44v6, CD44v810 and EpCAM combined with elevated CA19-9 had a lower RFS when compared with other groups $(p=0.004)$ (Fig. 8). 


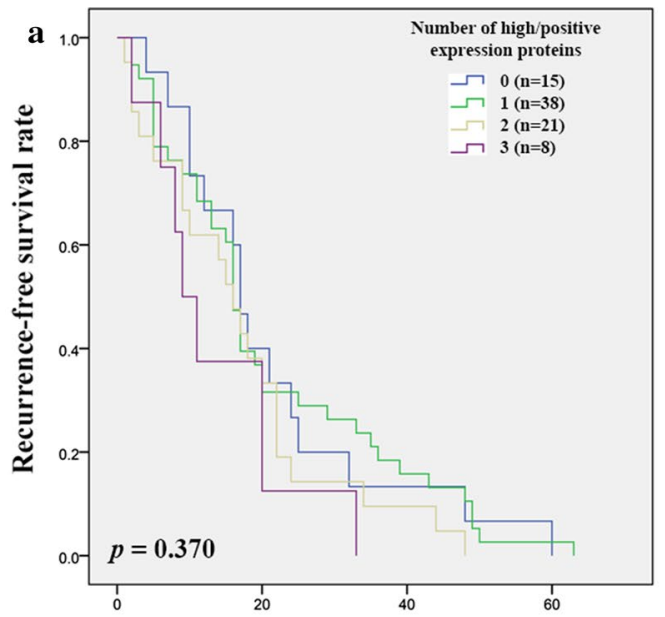

Follow-up period (months)

b

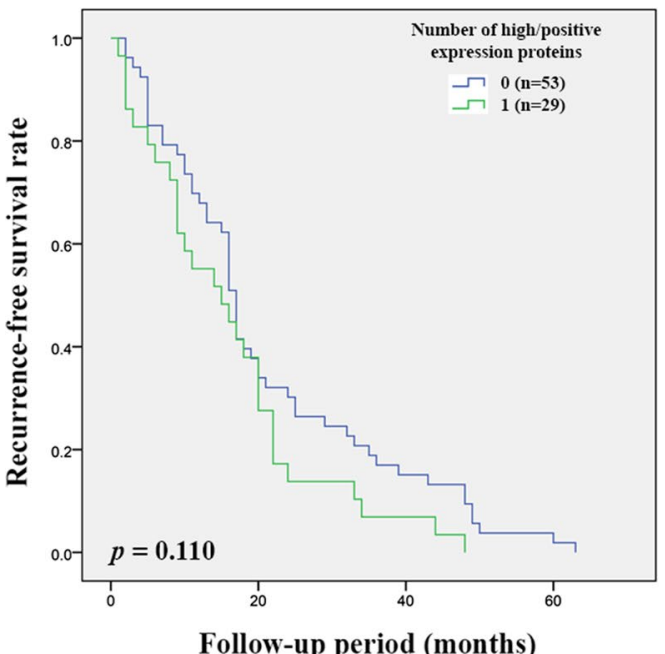

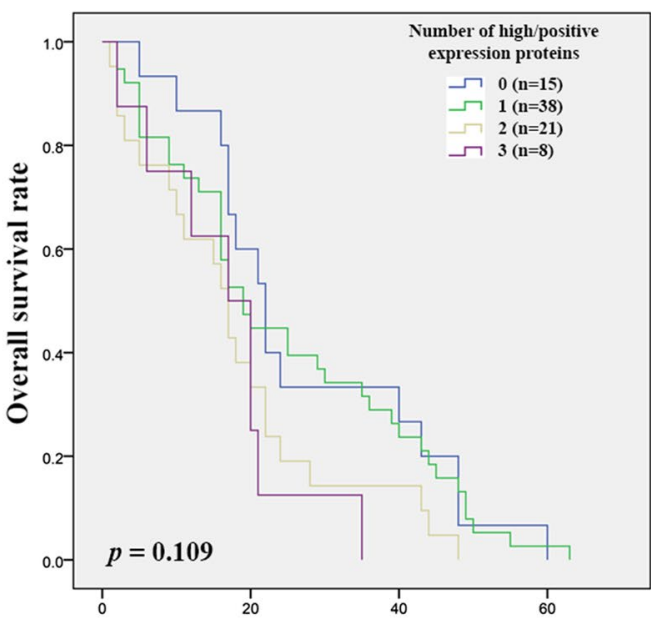

Follow-up period (months)

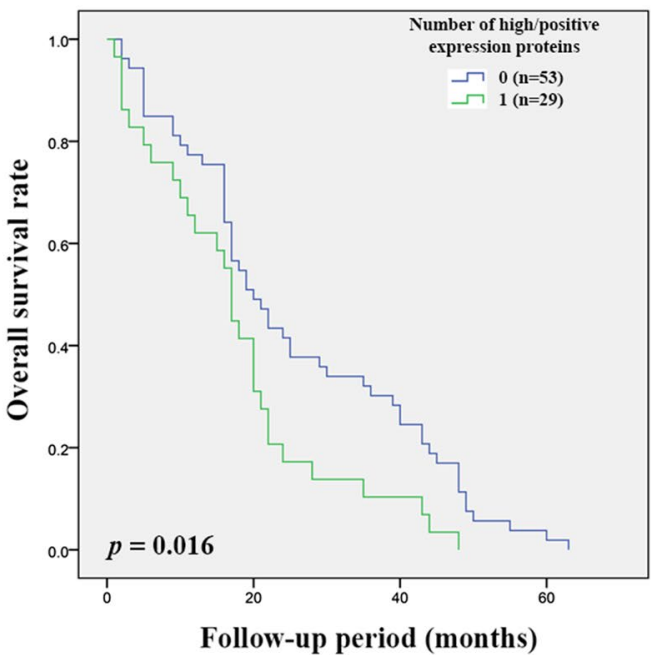

Fig. 6 Kaplan-Meier analyses of recurrence-free and overall survival on the combination of CD44, CD44v6, and CD44v8-10 expression in extrahepatic CCA patients. a Recurrence-free and overall survival of patients according to the number of high or positive expression proteins. $\mathbf{b}$ Recurrence-free and overall survival of patients with zero or one high or positive expression marker versus high or positive expression of two or three markers

\section{Discussion}

CCA is a malignant tumor with an asymptomatic early stage so that the disease is usually diagnosed once it has become advanced, resulting in a poor outcome for patients after treatment [23]. Even though several therapeutic approaches can be considered for CCA treatment, the recurrence rate is still high and leads to a high mortality in CCA patients [5]. Many studies suggest that tumor size and metastatic status are potential factors influencing RFS and OS in CCA patients [24-27]. Similar to our study, we found that CCA patients with a high primary tumor stage, presence of regional lymph node metastasis and high TNM staging have a lower RFS and OS compared with other groups of patients. Even though several studies have reported potential pathological factors for predicting CCA recurrence, effective biomarkers are required to assess the potential outcome of patients, including survival rate and the probability of recurrence after treatment. Moreover, the presence of such markers is likely to be useful for targeted therapy in order to prevent cancer progression and recurrence.

The subpopulation of cancer cells with stem cell-like properties, CSCs, has been reported to be involved in many cancer processes such as tumor growth, metastasis, resistance to treatment, as well as recurrence [28]. Raggi et al. demonstrated the existent of CSC in biliary tract cancer (BTC) and suggest that the isolated BTC cells that express CD24, CD44 or EpCAM had a higher potential of 

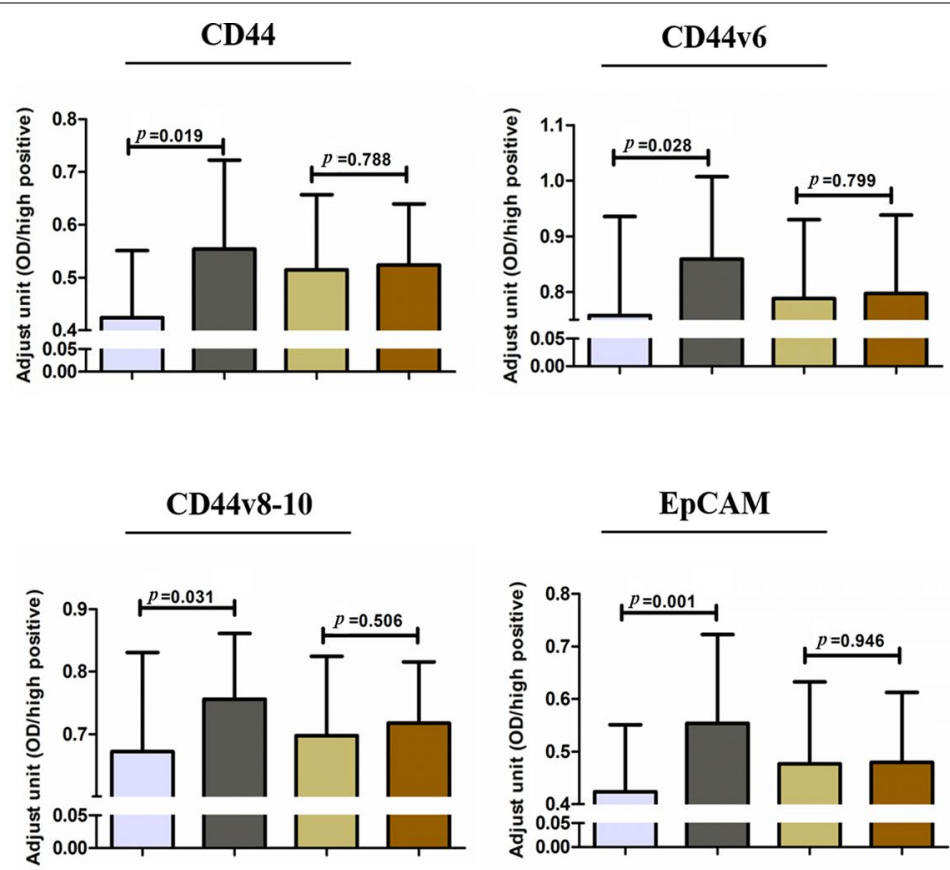

Fig. 7 The different levels of soluble CD44, CD44v6, CD44v8-10, and EpCAM in patients with and without recurrence according to tumor staging. Adjusted units were calculated from optical density (OD) sample/OD high positive sample

tumorigenesis than the negative groups [29]. In addition, other CSC markers have also been reported as markers for CSC in CCA [7]. Therefore, CSC markers might be used to predict CCA progression and recurrence. To answer this hypothesis, we performed immunohistochemical staining to evaluate the expression of 6 putative CSC markers, CD44, CD44v6, CD44v8-10, CD133, EpCAM and ALDH1A1 in CCA tissue. The results show that among the 6 CSC markers investigated, the expression of CD44 and its variant isoforms (CD44v6 and CD44v8-10), and also ALDH1A1, were associated with tumor progression and poor outcome of CCA patients, including short RFS and OS. CD44 is a well-known marker that plays an important role in tumor progression, but the different isoforms work differently [30]. There is considerable evidence suggesting that a high expression of CD44 is associated with tumor progression and recurrence [31,32], which is similar to the other two variant isoforms that have also been reported to be involved in cancer progression and recurrence [33-35]. This is consistent with our finding for CCA which shows that patients with a high expression of CD44, CD44v6, and CD44v8-10 had a shorter RFS and OS compared with the low expression group. In addition, the expression of these markers seems to increase along with tumor stage, suggesting that their expression is involved in tumor progression. ALDH1A1 is cytosolic enzyme that can convert retinol into retinoic acid. It plays an important role in many processes occurring in the normal cell, include growth, development and differentiation [21]. It has been reported to be marker for normal stem cells (SC) and also for CSC. Although many studies have reported that a high expression of ALDH1A1 is associated with tumor progression, this result is controversial as many studies have shown that a high expression of ALDH1A1 is correlated with a favorable prognosis in different cancers [21]. In the present study, we found that a high expression of ALDH1A1 was also associated with a favorable prognosis for CCA patients. There is evidence suggesting that a combination of protein expressions has more potential to divide patients into the different prognostic groups [36]. Thus, the correlation of our 4 promising markers was also analyzed. A significant positive correlation was found in CD44, CD44v6 and CD44v8-10, with the combination of high expression in two or three markers being more useful in dividing patients into the different prognostic groups. On the other hand, there was no correlation between ALDH1A1 and the other markers.

The panel of protein expression markers (CD44, CD44v6, and CD44v8-10) shows more efficacy in discriminating patients into different prognostic groups than the individual markers. Moreover, the elevation of these markers was also associated with RFS. Therefore, we further investigated the levels of these markers in the serum using the ELISA technique so that it can be used diagnostically for predicting factors for CCA recurrence. 
Table 6 The correlation between CSC marker levels in sera with clinicopathological features and laboratory results in early stage CCA patients

\begin{tabular}{|c|c|c|c|c|c|c|c|c|c|}
\hline \multirow[t]{2}{*}{ Variables } & \multicolumn{9}{|c|}{ Early stage (TNM stage I, II) } \\
\hline & $\mathrm{n}$ & CD44 & $p$ & CD44v6 & $p$ & CD44v8-10 & $p$ & EpCAM & $p$ \\
\hline \multicolumn{10}{|l|}{ Sex } \\
\hline Female & 22 & $0.47 \pm 0.13$ & & $0.80 \pm 0.17$ & & $0.70 \pm 0.13$ & & $0.46 \pm 0.16$ & \\
\hline Male & 38 & $0.51 \pm 0.16$ & 0.306 & $0.79 \pm 0.18$ & 0.861 & $0.70 \pm 0.15$ & 0.913 & $0.48 \pm 0.15$ & 0.623 \\
\hline \multicolumn{10}{|l|}{ Age (year) } \\
\hline Less than 61 & 30 & $0.51 \pm 0.15$ & & $0.82 \pm 0.15$ & & $0.72 \pm 0.12$ & & $0.50 \pm 0.15$ & \\
\hline 61 or greater & 30 & $0.49 \pm 0.16$ & 0.579 & $0.77 \pm 0.20$ & 0.220 & $0.68 \pm 0.17$ & 0.276 & $0.44 \pm 0.16$ & 0.102 \\
\hline \multicolumn{10}{|l|}{ Tumor site } \\
\hline Intrahepatic & 31 & $0.50 \pm 0.13$ & & $0.80 \pm 0.15$ & & $0.71 \pm 0.13$ & & $0.47 \pm 0.13$ & \\
\hline Extrahepatic & 29 & $0.49 \pm 0.18$ & 0.888 & $0.79 \pm 0.20$ & 0.778 & $0.70 \pm 0.17$ & 0.710 & $0.47 \pm 0.18$ & 0.928 \\
\hline \multicolumn{10}{|l|}{ Histology type } \\
\hline Papillary & 38 & $0.52 \pm 0.13$ & & $0.82 \pm 0.14$ & & $0.72 \pm 0.13$ & & $0.48 \pm 0.16$ & \\
\hline Others & 22 & $0.47 \pm 0.18$ & 0.229 & $0.74 \pm 0.21$ & 0.079 & $0.68 \pm 0.17$ & 0.277 & $0.45 \pm 0.16$ & 0.449 \\
\hline \multicolumn{10}{|l|}{ Tumor marker } \\
\hline \multicolumn{10}{|c|}{ CA19-9 (U/mL) } \\
\hline$<37$ & 24 & $0.44 \pm 0.13$ & & $0.77 \pm 0.16$ & & $0.67 \pm 0.14$ & & $0.40 \pm 0.11$ & \\
\hline$\geq 37$ & 21 & $0.57 \pm 0.15$ & 0.006 & $0.85 \pm 0.13$ & 0.072 & $0.77 \pm 0.10$ & 0.011 & $0.54 \pm 0.14$ & $<0.001$ \\
\hline \multicolumn{10}{|l|}{ CEA (ng/mL) } \\
\hline$<2.5$ & 16 & $0.48 \pm 0.12$ & & $0.81 \pm 0.15$ & & $0.70 \pm 0.14$ & & $0.44 \pm 0.12$ & \\
\hline$\geq 2.5$ & 30 & $0.52 \pm 0.16$ & 0.405 & $0.81 \pm 0.15$ & 0.937 & $0.72 \pm 0.13$ & 0.653 & $0.48 \pm 0.15$ & 0.286 \\
\hline \multicolumn{10}{|l|}{ AFP $(I U / m L)$} \\
\hline$<5$ & 33 & $0.48 \pm 0.15$ & & $0.79 \pm 0.14$ & & $0.70 \pm 0.13$ & & $0.45 \pm 0.14$ & \\
\hline$\geq 5$ & 4 & $0.55 \pm 0.21$ & 0.387 & $0.80 \pm 0.14$ & 0.894 & $0.71 \pm 0.13$ & 0.937 & $0.50 \pm 0.21$ & 0.553 \\
\hline \multicolumn{10}{|c|}{ Liver function test } \\
\hline \multicolumn{10}{|c|}{ Direct bilirubin (mg/dL) } \\
\hline$<1.5$ & 35 & $0.48 \pm 0.14$ & & $0.79 \pm 0.15$ & & $0.69 \pm 0.13$ & & $0.44 \pm 0.13$ & \\
\hline$\geq 1.5$ & 18 & $0.53 \pm 0.19$ & 0.261 & $0.79 \pm 0.22$ & 0.937 & $0.71 \pm 0.18$ & 0.600 & $0.49 \pm 0.17$ & 0.276 \\
\hline \multicolumn{10}{|c|}{ Total bilirubin (mg/dL) } \\
\hline$<2.5$ & 33 & $0.49 \pm 0.14$ & & $0.80 \pm 0.15$ & & $0.70 \pm 0.14$ & & $0.46 \pm 0.13$ & \\
\hline$\geq 2.5$ & 22 & $0.51 \pm 0.18$ & 0.571 & $0.78 \pm 0.21$ & 0.696 & $0.70 \pm 0.17$ & 0.866 & $0.46 \pm 0.17$ & 0.992 \\
\hline \multicolumn{10}{|l|}{ ALT (U/L) } \\
\hline$<40$ & 30 & $0.50 \pm 0.14$ & & $0.81 \pm 0.16$ & & $0.73 \pm 0.12$ & & $0.46 \pm 0.11$ & \\
\hline$\geq 40$ & 23 & $0.48 \pm 0.18$ & 0.521 & $0.75 \pm 0.19$ & 0.238 & $0.66 \pm 0.17$ & 0.089 & $0.46 \pm 0.19$ & 0.888 \\
\hline \multicolumn{10}{|l|}{ AST (U/L) } \\
\hline$<40$ & 28 & $0.48 \pm 0.13$ & & $0.79 \pm 0.16$ & & $0.70 \pm 0.13$ & & $0.47 \pm 0.13$ & \\
\hline$\geq 40$ & 25 & $0.50 \pm 0.18$ & 0.709 & $0.78 \pm 0.19$ & 0.794 & $0.69 \pm 0.17$ & 0.752 & $0.46 \pm 0.17$ & 0.834 \\
\hline \multicolumn{10}{|l|}{$\mathrm{ALP}(\mathrm{U} / \mathrm{L})$} \\
\hline$<130$ & 18 & $0.45 \pm 0.15$ & & $0.76 \pm 0.16$ & & $0.68 \pm 0.13$ & & $0.42 \pm 0.12$ & \\
\hline$\geq 130$ & 35 & $0.52 \pm 0.16$ & 0.129 & $0.80 \pm 0.18$ & 0.479 & $0.71 \pm 0.16$ & 0.575 & $0.48 \pm 0.16$ & 0.151 \\
\hline
\end{tabular}

CD44 cluster of differentiation 44, CD44v CD44 variant, EpCAM epithelial cell adhesion molecules, TNM primary tumor-node-metastasis, CA19-9 cancer antigen 19-9, $C E A$ carcinoembryonic antigen, AFP alpha-fetoprotein, $A L T$ alanine aminotransferase, AST aspartate aminotransferase, $A L P$ alkaline phosphatase

As many studies suggest that soluble EpCAM is associated with an aggressive tumor phenotype [18-20], soluble EpCAM was also considered to be a marker for CCA recurrence. According to the literature, tumor staging is an important factor involved in tumor recurrence in CCA patients [27], and our results on IHC also demonstrate that tumor staging has the potential to predict CCA recurrence. Therefore, in order to determine the effect of staging on cancer recurrence, the different levels of soluble CD44, CD44v6, CD44v8-10 and EpCAM in patients with and without recurrence were examined according to staging. The results indicate that 
Table 7 The correlation between CSC marker levels in sera with clinicopathological features and laboratory results in late stage CCA patients

\begin{tabular}{|c|c|c|c|c|c|c|c|c|c|}
\hline \multirow[t]{2}{*}{ Variables } & \multicolumn{9}{|c|}{ Late stage (TNM stage III, IV) } \\
\hline & $\mathrm{n}$ & CD44 & $p$ & CD44v6 & $p$ & CD44v8-10 & $p$ & EpCAM & $p$ \\
\hline \multicolumn{10}{|l|}{ Sex } \\
\hline Female & 24 & $0.51 \pm 0.16$ & & $0.80 \pm 0.15$ & & $0.71 \pm 0.12$ & & $0.50 \pm 0.17$ & \\
\hline Male & 43 & $0.52 \pm 0.12$ & 0.584 & $0.78 \pm 0.14$ & 0.573 & $0.70 \pm 0.12$ & 0.940 & $0.47 \pm 0.13$ & 0.435 \\
\hline \multicolumn{10}{|l|}{ Age (year) } \\
\hline Less than 61 & 32 & $0.51 \pm 0.12$ & & $0.80 \pm 0.12$ & & $0.69 \pm 0.10$ & & $0.46 \pm 0.14$ & \\
\hline 61 or greater & 35 & $0.52 \pm 0.14$ & 0.769 & $0.78 \pm 0.16$ & 0.651 & $0.71 \pm 0.13$ & 0.505 & $0.50 \pm 0.16$ & 0.289 \\
\hline \multicolumn{10}{|l|}{ Tumor site } \\
\hline Intrahepatic & 38 & $0.50 \pm 0.13$ & & $0.77 \pm 0.15$ & & $0.70 \pm 0.11$ & & $0.46 \pm 0.12$ & \\
\hline Extrahepatic & 29 & $0.54 \pm 0.13$ & 0.231 & $0.82 \pm 0.13$ & 0.128 & $0.71 \pm 0.13$ & 0.539 & $0.50 \pm 0.17$ & 0.259 \\
\hline \multicolumn{10}{|l|}{ Histology type } \\
\hline Papillary & 29 & $0.51 \pm 0.14$ & & $0.78 \pm 0.15$ & & $0.70 \pm 0.12$ & & $0.47 \pm 0.17$ & \\
\hline Others & 38 & $0.53 \pm 0.13$ & 0.599 & $0.80 \pm 0.14$ & 0.510 & $0.71 \pm 0.12$ & 0.809 & $0.49 \pm 0.13$ & 0.562 \\
\hline \multicolumn{10}{|l|}{ Tumor marker } \\
\hline \multicolumn{10}{|c|}{ CA19-9 (U/mL) } \\
\hline$<37$ & 13 & $0.47 \pm 0.14$ & & $0.79 \pm 0.13$ & & $0.71 \pm 0.13$ & & $0.44 \pm 0.13$ & \\
\hline$\geq 37$ & 33 & $0.53 \pm 0.13$ & 0.152 & $0.80 \pm 0.16$ & 0.828 & $0.70 \pm 0.11$ & 0.841 & $0.48 \pm 0.13$ & 0.257 \\
\hline \multicolumn{10}{|l|}{ CEA (ng/mL) } \\
\hline$<2.5$ & 6 & $0.51 \pm 0.20$ & & $0.71 \pm 0.19$ & & $0.71 \pm 0.10$ & & $0.52 \pm 0.09$ & \\
\hline$\geq 2.5$ & 37 & $0.51 \pm 0.11$ & 0.888 & $0.80 \pm 0.13$ & 0.171 & $0.69 \pm 0.10$ & 0.598 & $0.44 \pm 0.11$ & 0.108 \\
\hline \multicolumn{10}{|l|}{ AFP $(I U / m L)$} \\
\hline$<5$ & 33 & $0.52 \pm 0.14$ & & $0.79 \pm 0.16$ & & $0.70 \pm 0.11$ & & $0.46 \pm 0.11$ & \\
\hline$\geq 5$ & 4 & $0.45 \pm 0.20$ & 0.357 & $0.77 \pm 0.11$ & 0.792 & $0.61 \pm 0.06$ & 0.118 & $0.38 \pm 0.11$ & 0.199 \\
\hline \multicolumn{10}{|c|}{ Liver function test } \\
\hline \multicolumn{10}{|c|}{ Direct bilirubin (mg/dL) } \\
\hline$<1.5$ & 39 & $0.50 \pm 0.14$ & & $0.77 \pm 0.16$ & & $0.70 \pm 0.11$ & & $0.46 \pm 0.13$ & \\
\hline$\geq 1.5$ & 17 & $0.56 \pm 0.12$ & 0.137 & $0.85 \pm 0.09$ & 0.029 & $0.71 \pm 0.11$ & 0.632 & $0.51 \pm 0.14$ & 0.227 \\
\hline \multicolumn{10}{|c|}{ Total bilirubin (mg/dL) } \\
\hline$<2.5$ & 35 & $0.49 \pm 0.14$ & & $0.76 \pm 0.16$ & & $0.68 \pm 0.11$ & & $0.46 \pm 0.13$ & \\
\hline$\geq 2.5$ & 21 & $0.55 \pm 0.12$ & 0.089 & $0.85 \pm 0.11$ & 0.037 & $0.73 \pm 0.11$ & 0.149 & $0.51 \pm 0.13$ & 0.181 \\
\hline \multicolumn{10}{|l|}{ ALT (U/L) } \\
\hline$<40$ & 28 & $0.49 \pm 0.14$ & & $0.76 \pm 0.16$ & & $0.70 \pm 0.12$ & & $0.48 \pm 0.14$ & \\
\hline$\geq 40$ & 28 & $0.54 \pm 0.12$ & 0.148 & $0.83 \pm 0.13$ & 0.091 & $0.70 \pm 0.11$ & 0.946 & $0.47 \pm 0.12$ & 0.708 \\
\hline \multicolumn{10}{|l|}{ AST (U/L) } \\
\hline$<40$ & 25 & $0.49 \pm 0.15$ & & $0.75 \pm 0.18$ & & $0.68 \pm 0.11$ & & $0.45 \pm 0.12$ & \\
\hline$\geq 40$ & 31 & $0.54 \pm 0.12$ & 0.150 & $0.83 \pm 0.11$ & 0.037 & $0.72 \pm 0.11$ & 0.187 & $0.49 \pm 0.14$ & 0.295 \\
\hline \multicolumn{10}{|l|}{$\mathrm{ALP}(\mathrm{U} / \mathrm{L})$} \\
\hline$<130$ & 19 & $0.47 \pm 0.13$ & & $0.74 \pm 0.16$ & & $0.65 \pm 0.11$ & & $0.41 \pm 0.11$ & \\
\hline$\geq 130$ & 37 & $0.54 \pm 0.13$ & 0.056 & $0.82 \pm 0.13$ & 0.049 & $0.72 \pm 0.11$ & 0.024 & $0.51 \pm 0.13$ & 0.006 \\
\hline
\end{tabular}

CD44 cluster of differentiation 44, CD44v CD44 variant, EpCAM epithelial cell adhesion molecules, TNM primary tumor-node-metastasis, CA19-9 cancer antigen 19-9, $C E A$ carcinoembryonic antigen, AFP alpha-fetoprotein, ALT alanine aminotransferase, AST aspartate aminotransferase, ALP: alkaline phosphatase

early stage tumors are less variable than late or advanced stage tumors. Thus, the recurrence of cancer is caused by the inherent resistance of cancer cells [37]. Our results on early stage CCA patients show that patients with a low $\mathrm{T}$ stage, absence of lymph node involvement and no distant metastases but with recurrence had higher soluble levels of CD44, CD44v6, CD44v8-10 and EpCAM compared with those patients without recurrence. Accumulating evidence indicates that highly proliferative cancer cells can be killed by chemotherapy and radiotherapy, however a subpopulation of cancer cells with therapeutic resistance might survive and lead to relapse [6]. CD44 is known as a surface marker associated with CSC in various cancer types, and several CD44 variant 
Table 8 Predictive values for soluble CD44, CD44v6, CD44v8-10 and EpCAM for predicting CCA recurrence using the optimal cut-off derived from the ROC curve in early stage of CCA patients

\begin{tabular}{llllllll}
\hline Variables & AUC $(\mathbf{9 5} \% \mathbf{C l})$ & Cut-off & Sensitivity (\%) & Specificity (\%) & PPV (\%) & NPV (\%) & $\boldsymbol{p}$ \\
\hline CD44 & $0.670(0.521-0.819)$ & 0.505 & 68.0 & 63.0 & 51.7 & 77.4 & 0.029 \\
CD44v6 & $0.670(0.529-0.811)$ & 0.814 & 68.0 & 68.0 & 55.6 & 78.8 \\
CD44v8-10 & $0.702(0.563-0.841)$ & 0.713 & 73.0 & 71.0 & 59.3 & 81.8 \\
EpCAM & $0.739(0.598-0.880)$ & 0.506 & 68.0 & 76.0 & 62.5 & 80.6 & 0.029 \\
\hline
\end{tabular}

CD44 cluster of differentiation 44, CD44v CD44 variant, EpCAM epithelial cell adhesion molecules, AUC area under curve, 95\% CI 95\% confidence interval, $P P V$ positive predictive value, NPV negative predictive value

Table 9 Predictive risk of CCA recurrence in early stage patients using soluble CD44, CD44v6, CD44v8-10, and EpCAM

\begin{tabular}{|c|c|c|c|c|}
\hline \multirow[t]{2}{*}{ Comparative prediction } & \multicolumn{4}{|c|}{ Post-operative recurrence } \\
\hline & OR crude & $p(95 \% \mathrm{Cl})$ & OR adjusted & $p(95 \% \mathrm{Cl})$ \\
\hline CD44 $\geq 0.505$ vs. $<0.505$ & 3.67 & $0.022(1.207-11.183)$ & 3.62 & $0.031(1.126-11.66)$ \\
\hline$C D 44 v 6 \geq 0.814$ vs. $<0.814$ & 4.64 & $0.008(1.503-14.346)$ & 4.98 & $0.009(1.504-16.473)$ \\
\hline CD44v8-10 $\geq 0.713$ vs. $<0.713$ & 6.55 & $0.002(2.029-21.116)$ & 5.92 & $0.004(1.779-19.706)$ \\
\hline EpCAM $\geq 0.814$ vs. $<0.814$ & 6.91 & $0.001(2.147-22.202)$ & 6.23 & $0.003(1.867-20.793)$ \\
\hline
\end{tabular}

CD44 cluster of differentiation 44, CD44v CD44 variant, EpCAM epithelial cell adhesion molecules, OR odds ratio, OR adjusted odds ratio adjusted for age and sex statistical analysis, $95 \% \mathrm{Cl}$ 95\% confidence interval

Table 10 Predictive risk of CCA recurrence in early stage patients using either the combination of soluble CSC markers, CD44, CD44v6, CD44v8-10 and EpCAM or the combination of soluble CSC markers with CA19-9

\begin{tabular}{|c|c|c|c|c|}
\hline Comparative prediction & $\begin{array}{l}\text { OR } \\
\text { Crude }\end{array}$ & $\begin{array}{l}p \\
(95 \% \mathrm{Cl})\end{array}$ & $\begin{array}{l}\text { OR } \\
\text { Adjusted }\end{array}$ & $\begin{array}{l}p \\
(95 \% \mathrm{Cl})\end{array}$ \\
\hline \multicolumn{5}{|l|}{ Post-operative recurrence } \\
\hline \multicolumn{5}{|l|}{ Levels of all markers $\geq$ cut-offa $^{a}$} \\
\hline $\mathrm{No}^{\mathrm{b}}(46)$ & 1 & 0.004 & 1 & 0.006 \\
\hline Yes (14) & 7.08 & $(1.867-26.870)$ & 7.39 & $(1.760-31.071)$ \\
\hline \multicolumn{5}{|c|}{ CA19-9 $\geq 37 \mathrm{U} / \mathrm{mL}+$ Levels of all markers $\geq$ cut-off } \\
\hline $\mathrm{No}+\mathrm{No}$ and $\mathrm{Yes}+\mathrm{No}$ and $\mathrm{No}+\mathrm{Yes}(36)$ & 1 & 0.005 & 1 & 0.011 \\
\hline Yes + Yes (9) & 12.25 & $(2.114-70.986)$ & 15.28 & $(1.879-124.320)$ \\
\hline
\end{tabular}

OR odds ratio, OR adjusted odds ratio adjusted for age and sex statistical analysis, $95 \%$ Cl 95\% confidence interval

a Levels of all markers: levels of CD44, CD44v6, CD44v8-10 and EpCAM

b No: patients with at least one of marker lower than a designated cut-off

isoforms are generated by alternative splicing processes [38]. Shi et al. reported that the expression of CD44v6 is up-regulated in the recurrence ovarian cancer, and this is also associated with cancer progression and metastasis [34]. Another CSC marker, CD44v8-10 stabilizes xCT, which is a cystine-glutamate transporter inducing glutathione synthesis. This process contributes to the tumor cells becoming resistant to oxidative stress, including reactive oxygen species (ROS) [39]. In addition, a study by Tayama et al. on ovarian cancer demonstrated that chemotherapy mostly eliminated the EpCAM-negative population compared with the EpCAM-positive population, suggesting that the EpCAM-positive population contributes to chemoresistance and cancer recurrence after chemotherapy [40]. Thus, the CSC markers, CD44, CD44v6, CD44v8-10, and EpCAM have the potential to predict cancer recurrence including CCA.

The levels of tumor markers (CA19-9, CEA, and AFP) and a liver function test were also used to monitor CCA patients after treatment [27]. In this study, we also found that high levels of soluble CD44, CD44v6, CD44v810 and EpCAM were correlated with elevated levels of CA19-9, suggesting that their expression is involved in tumor progression. However, in late stage disease, there was no difference in the levels of soluble CD44, CD44v6, CD44v8-10 and EpCAM in patients with and without 


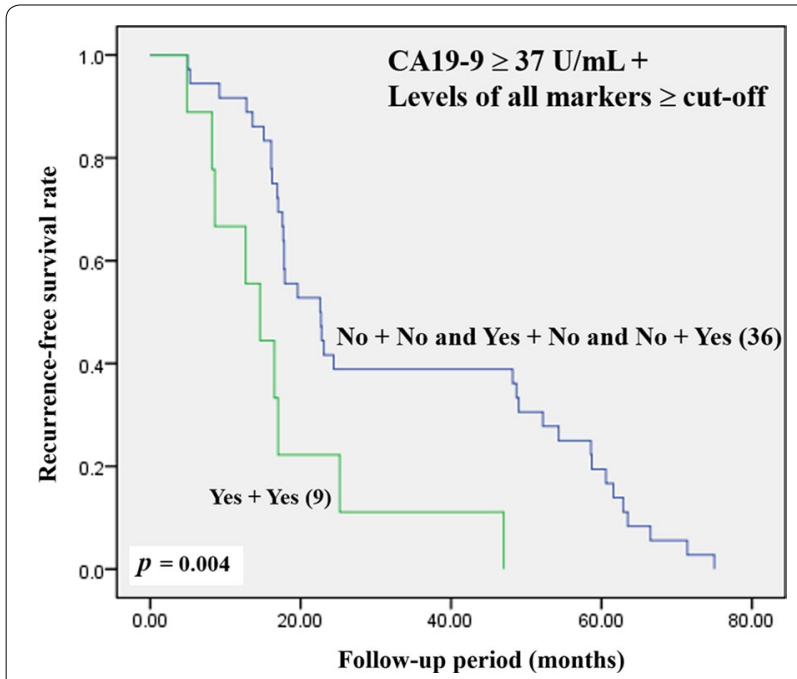

Fig. 8 Kaplan-Meier analysis of recurrence-free survival on patients with high levels of CD44, CD44v6, CD44v8-10 and EpCAM with CA19-9 versus other groups of patients

\section{Supplementary information}

Supplementary information accompanies this paper at https://doi. org/10.1186/s12967-020-02243-w.

Additional file 1: Table S1. The detailed information of sera CCA samples. Additional file 2: Fig S1. ROC curve of soluble CD44, CD44v6, CD44v8-10, and EpCAM for predicting CCA recurrence in early stage CCA patients. AUC represents the area under curve for each protein.

Additional file 3: Fig S2. ROC curve of soluble CD44, CD44v6, CD44v8-10, and EpCAM for predicting CCA recurrence in late stage CCA patients. AUC represents the area under curve for each protein.

\section{Abbreviations}

ALDH1A1: Aldehyde dehydrogenase 1A1; CCA: Cholangiocarcinoma; CSC: Cancer stem cell; CD44: Cluster of differentiation 44; CD44v6: Cluster of differentiation 44 variant 6; CD44v8-10: Cluster of differentiation 44 variants 8-10; CD133: Cluster of differentiation 133; EpCAM: Epithelial cell adhesion molecule; CA19-9: Carbohydrate antigen; CEA: Carcinoembryonic antigen; OS: Overall survival; RFS: Recurrence-free survival.

\section{Acknowledgements}

We thank Professor Trevor N. Petney for editing the MS via the Publication Clinic KKU, Thailand.

\section{Authors' contributions}

$\mathrm{SP}, \mathrm{WL}$, and NN were responsible for the experimental design. SP wrote the manuscript. SP and MT evaluated IHC, ELISA of the specimens, performed the statistical analyses. NK and AT are surgeons participating to tumor surgery and clinicopathological data collection. WL, NN and HS revised the first draft of the manuscript. All authors read and approved the final manuscript.

\section{Funding}

The study was supported by funding organizations include The Thailand Research Fund (Royal Golden Jubilee Ph.D. Program) and Khon Kaen University (Grant No. PHD/0084/2560) allocated to WL and SP. Invitation Research Grant (IN62142) allocated to SP. A grant from Cholangiocarcinoma Screening and Care Program (CASCAP-09), a Grant from the Thailand Research Fund (Grant No. RSA5980013) allocated to WL.

\section{Availability of data and materials}

The datasets generated during and/or analyzed during the current study are available from the corresponding author on reasonable request.

\section{Ethics approval and consent to participate}

This study was approved by the Human Research Ethics Committees, Khon Kaen University, Thailand (HE611412).

\section{Consent for publication}

Not applicable.

\section{Competing interests}

The authors declare that they have no competing interests.

\section{Author details}

1 Department of Biochemistry, Faculty of Medicine, Khon Kaen University, 123 Mittraparp Road, Muang District, Khon Kaen 40002, Thailand. ${ }^{2}$ Cholangiocarcinoma Screening and Care Program (CASCAP), Khon Kaen University, Khon Kaen 40002, Thailand. ${ }^{3}$ Cholangiocarcinoma Research Institute, Faculty of Medicine, Khon Kaen University, Khon Kaen 40002, Thailand. ${ }^{4}$ Department of Surgery, Faculty of Medicine, Khon Kaen University, Khon Kaen 40002, Thailand. ${ }^{5}$ Department of Pathology, Faculty of Medicine, Khon Kaen University, Khon Kaen 40002, Thailand. ${ }^{6}$ Division of Gene Regulation, Institute for Advanced Medical Research (IAMR), Keio University School of Medicine, Tokyo 160-8582, Japan.

Received: 28 September 2019 Accepted: 27 January 2020

Published online: 10 February 2020 


\section{References}

1. Ghouri YA, Mian I, Blechacz B. Cancer review: cholangiocarcinoma. J Carcinog. 2015;14:1

2. Blechacz B. Cholangiocarcinoma: current Knowledge and New Developments. Gut Liver. 2017:11:13-26.

3. Titapun A, Pugkhem A, Luvira V, SrisukT, Somintara O, Saeseow OT, et al. Outcome of curative resection for perihilar cholangiocarcinoma in Northeast Thailand. World J Gastrointest Oncol. 2015;7:503-12.

4. Hoehn RS, Wima K, Ertel AE, Meier A, Ahmad SA, Shah SA, et al. Adjuvant chemotherapy and radiation therapy is associated with improved survival for patients with extrahepatic cholangiocarcinoma. Ann Surg Oncol. 2015;22(Suppl 3):S1133-9.

5. Luvira V, Eurboonyanun C, Bhudhisawasdi V, Pugkhem A, Pairojkul C, Luvira $V$, et al. Patterns of recurrence after resection of mass-forming type intrahepatic cholangiocarcinomas. Asian Pac J Cancer Prev. 2016;17:4735-9.

6. Peitzsch C, Tyutyunnykova A, Pantel K, Dubrovska A. Cancer stem cells: the root of tumor recurrence and metastases. Semin Cancer Biol. 2017:44:10-24.

7. Mayr C, Ocker M, Ritter M, Pichler M, Neureiter D, Kiesslich T. Biliary tract cancer stem cells — translational options and challenges. World J Gastroenterol. 2017;23:2470-82

8. Guo Z, Li LQ, Jiang JH, Ou C, Zeng LX, Xiang BD. Cancer stem cell markers correlate with early recurrence and survival in hepatocellular carcinoma. World J Gastroenterol. 2014;20:2098-106.

9. Kakehashi A, Ishii N, Sugihara E, Gi M, Saya H, Wanibuchi H. CD44 variant 9 is a potential biomarker of tumor initiating cells predicting survival outcome in hepatitis C virus-positive patients with resected hepatocellular carcinoma. Cancer Sci. 2016;107:609-18.

10. Miyoshi Y, Shien T, Ogiya A, Ishida N, Yamazaki K, Horii R, et al. Differences in expression of the cancer stem cell marker aldehyde dehydrogenase 1 among estrogen receptor-positive/human epidermal growth factor receptor type 2-negative breast cancer cases with early, late, and no recurrence. Breast Cancer Res. 2016;18:73.

11. Thanee M, Loilome W, Techasen A, Sugihara E, Okazaki S, Abe S, et al. CD44 variant-dependent redox status regulation in liver fluke-associated cholangiocarcinoma: a target for cholangiocarcinoma treatment. Cancer Sci. 2016;107:991-1000.

12. Liu K, Hao M, Ouyang Y, Zheng J, Chen D. CD133(+) cancer stem cells promoted by VEGF accelerate the recurrence of hepatocellular carcinoma. Sci Rep. 2017;7:41499.

13. Senbanjo LT, Chellaiah MA. CD44: a multifunctional cell surface adhesion receptor is a regulator of progression and metastasis of cancer cells. Front Cell Dev Biol. 2017:5:18.

14. Katoh S, Goi T, Naruse T, Ueda Y, Kurebayashi H, Nakazawa T, et al. Cancer stem cell marker in circulating tumor cells: expression of CD44 variant exon 9 is strongly correlated to treatment refractoriness, recurrence and prognosis of human colorectal cancer. Anticancer Res. 2015;35:239-44.

15. Chen S, Song X, Chen Z, Li X, Li M, Liu H, et al. CD133 expression and the prognosis of colorectal cancer: a systematic review and meta-analysis. PLOS ONE. 2013:8:e56380.

16. Wang MH, Sun R, Zhou XM, Zhang MY, Lu JB, Yang Y, et al. Epithelial cell adhesion molecule overexpression regulates epithelial-mesenchymal transition, stemness and metastasis of nasopharyngeal carcinoma cells via the PTEN/AKT/mTOR pathway. Cell Death Dis. 2018;9:2.

17. Schnell U, Cirulli V, Giepmans BN. EpCAM: structure and function in health and disease. Biochem Biophys Acta. 2013;1828:1989-2001.

18. Lin CW, Liao MY, Lin WW, Wang YP, LuTY, Wu HC. Epithelial cell adhesion molecule regulates tumor initiation and tumorigenesis via activating reprogramming factors and epithelial-mesenchymal transition gene expression in colon cancer. J Biol Chem. 2012;287:39449-59.

19. Kuan II, Liang KH, Wang YP, Kuo TW, Meir YJ, Wu SC, et al. EpEX/EpCAM and Oct4 or Klf4 alone are sufficient to generate induced pluripotent stem cells through STAT3 and HIF2alpha. Sci Rep. 2017:7:41852.

20. Liang KH, Tso HC, Hung SH, Kuan II, Lai JK, Ke FY, et al. Extracellular domain of EpCAM enhances tumor progression through EGFR signaling in colon cancer cells. Cancer Lett. 2018;433:165-75.

21. Tomita H, Tanaka K, Tanaka T, Hara A. Aldehyde dehydrogenase $1 \mathrm{~A} 1$ in stem cells and cancer. Oncotarget. 2016;7:11018-32.

22. Namwat N, Puetkasichonpasutha J, Loilome W, Yongvanit P, Techasen A, Puapairoj A, et al. Downregulation of reversion-inducing-cysteine-rich protein with Kazal motifs (RECK) is associated with enhanced expression of matrix metalloproteinases and cholangiocarcinoma metastases. J Gastroenterol. 2011:46:664-75.

23. Banales JM, Cardinale V, Carpino G, Marzioni M, Andersen JB, Invernizzi P, et al. Expert consensus document: cholangiocarcinoma: current knowledge and future perspectives consensus statement from the European Network for the Study of Cholangiocarcinoma (ENS-CCA). Nat Rev Gastroenterol Hepatol. 2016;13:261-80.

24. Hyder O, Hatzaras I, Sotiropoulos GC, Paul A, Alexandrescu S, Marques H, et al. Recurrence after operative management of intrahepatic cholangiocarcinoma. Surgery. 2013;153:811-8.

25. Gil E, Joh JW, Park HC, Yu Jl, Jung SH, Kim JM. Predictors and patterns of recurrence after curative liver resection in intrahepatic cholangiocarcinoma, for application of postoperative radiotherapy: a retrospective study. World J Surg Oncol. 2015;13:227.

26. Komaya K, Ebata T, Shirai K, Ohira S, Morofuji N, Akutagawa A, et al. Recurrence after resection with curative intent for distal cholangiocarcinoma. The British journal of surgery. 2017;104:426-33.

27. Wang C, Pang S, Si-Ma H, Yang N, Zhang H, Fu Y, et al. Specific risk factors contributing to early and late recurrences of intrahepatic cholangiocarcinoma after curative resection. World J Surg Oncol. 2019;17:2.

28. Chang JC. Cancer stem cells: role in tumor growth, recurrence, metastasis, and treatment resistance. Medicine. 2016;95:S20-5.

29. Wang M, Xiao J, Shen M, Yahong Y, Tian R, Zhu F, et al. Isolation and characterization of tumorigenic extrahepatic cholangiocarcinoma cells with stem cell-like properties. Int J Cancer. 2011;128:72-81.

30. Basakran NS. CD44 as a potential diagnostic tumor marker. Saudi Med J. 2015;36:273-9.

31. Jeong BJ, Liang ZL, Huang SM, Lim JS, Kim JM, Lee HJ. CD44 is associated with tumor recurrence and is an independent poor prognostic factor for patients with localized clear cell renal cell carcinoma after nephrectomy. Exp Ther Med. 2012;3:811-7.

32. Molejon MI, Tellechea Jl, Moutardier V, Gasmi M, Ouaissi M, Turrini O, et al. Targeting CD44 as a novel therapeutic approach for treating pancreatic cancer recurrence. Oncoscience. 2015:2:572-5.

33. Hirata K, Suzuki H, Imaeda H, Matsuzaki J, Tsugawa H, Nagano O, et al. CD44 variant 9 expression in primary early gastric cancer as a predictive marker for recurrence. Br J Cancer. 2013;109:379-86.

34. Shi J, Zhou Z, Di W, Li N. Correlation of CD44v6 expression with ovarian cancer progression and recurrence. BMC cancer. 2013;13:182.

35. Hagiwara M, Kikuchi E, Kosaka T, Mikami S, Saya H, Oya M. Variant isoforms of CD44 expression in upper tract urothelial cancer as a predictive marker for recurrence and mortality. Urol Oncol-Semin Ori. 2016;34:337.

36. Shang L, Liu HJ, Hao JJ, Jiang YY, Shi F, Zhang Y, et al. A panel of overexpressed proteins for prognosis in esophageal squamous cell carcinoma. PLOS ONE. 2014;9:e111045.

37. Leary M, Heerboth S, Lapinska K, Sarkar S. Sensitization of Drug Resistant Cancer Cells: a Matter of Combination Therapy. Cancers (Basel). 2018;10:483.

38. Thapa R, Wilson GD. The Importance of CD44 as a Stem Cell Biomarker and Therapeutic Target in Cancer. Stem cells international. 2016;2016:2087204

39. Ishimoto T, Nagano O, Yae T, Tamada M, Motohara T, Oshima H, et al. CD44 variant regulates redox status in cancer cells by stabilizing the $\mathrm{XCT}$ subunit of system $\times c(-)$ and thereby promotes tumor growth. Cancer Cell. 2011;19:387-400

40. Tayama S, Motohara T, Narantuya D, Li C, Fujimoto K, Sakaguchi I, et al. The impact of EpCAM expression on response to chemotherapy and clinical outcomes in patients with epithelial ovarian cancer. Oncotarget. 2017:8:44312-25

41. Wang JK, Hu HJ, Shrestha A, Ma WJ, Yang Q, Liu F, et al. Can preoperative and postoperative CA19-9 levels predict survival and early recurrence in patients with resectable hilar cholangiocarcinoma? Oncotarget. 2017:8:45335-44.

\section{Publisher's Note}

Springer Nature remains neutral with regard to jurisdictional claims in published maps and institutional affiliations. 